The Astronomical Journal, 120:1238-1264, 2000 September

(C) 2000. The American Astronomical Society. All rights reserved. Printed in U.S.A.

\title{
FORMATION OF A TIDAL DWARF GALAXY IN THE INTERACTING SYSTEM ARP 245 (NGC 2992/93)
}

\author{
P.-A. Duc, ${ }^{1,2}$ E. Brinks, ${ }^{3}$ V. Springel, ${ }^{4,5}$ B. Pichardo, ${ }^{6}$ P. Weilbacher, ${ }^{7}$ and I. F. Mirabel ${ }^{8,9}$ \\ Received 2000 February 29; accepted 2000 May 19
}

\begin{abstract}
Among the various phenomena observed in interacting galaxies is the ejection due to tidal forces of stellar and gaseous material into the intergalactic medium and its subsequent rearranging which can lead to the formation of self-gravitating tidal dwarf galaxies (TDGs). We investigate this process with a detailed multiwavelength study of the interacting system Arp 245 and a numerical model of the collision computed with a Tree-SPH code. Our observations consist of optical/near-infrared broadband imaging, $\mathrm{H} \alpha$ imaging, optical spectroscopy, $\mathrm{H}$ I VLA cartography and CO line mapping. The system, composed of the two spiral galaxies NGC 2992 and NGC 2993, is observed at an early stage of the interaction, about $100 \mathrm{Myr}$ after perigalacticon, though at a time when tidal tails have already developed. The VLA observations disclose a third partner to the interaction: an edge-on, flat galaxy, FGC 0938, which looks strikingly undisturbed and might just be falling toward the NGC 2992/93 system. Our H I map shows prominent counterparts to the optical tails. Whereas the stellar and gaseous components of the plume that originates from NGC 2992 match, the stellar and H I tails emanating from NGC 2993 have a different morphology. In particular, the $\mathrm{H}$ I forms a ring, a feature that has been successfully reproduced by our numerical simulations. The $\mathrm{H}$ I emission in the system as a whole peaks at the tip of the NGC 2992 tail where a gas reservoir of about $10^{9} M \odot$, about $60 \%$ of the $\mathrm{H}$ I toward NGC 2992, coincides with a star-forming optical condensation, A245N. The latter tidal object exhibits properties ranging between those of dwarf irregular galaxies (structural parameters, gas content, star formation rate) and those of spiral disks (metallicity, star formation efficiency, stellar population). Although it is likely, based on our analysis of the $\mathrm{H}$ I and model data cube, that A245N might become an independent dwarf galaxy, the dynamical evidence is still open to debate. Prompted by the questions raised for this particular object, we discuss some issues related to the definition and identification of TDGs and highlight some specific conditions which seem required to form them. Finally, we outline what is needed in terms of future numerical simulations in order to further our understanding of these objects.
\end{abstract}

Key words: galaxies: individual (Arp 245, NGC 2992, NGC 2993, FGC 0938) - galaxies: interactions methods: numerical

\section{INTRODUCTION}

Research activity in the field of interacting galaxies has increased quite dramatically over the last thirty years (see the recent very comprehensive review by Struck 1999). Galactic collisions trigger a number of phenomena, such as the inward transportation of gas from distances of up to kiloparsecs to the nucleus, which is thought to be an efficient means to fuel a central starburst or nuclear activity. The inverse process is the ejection of material into the intergalactic medium by tidal forces. The prominent tidal tails and bridges that emanate from interacting galaxies have

\footnotetext{
${ }^{1}$ CNRS URA 2052 and CEA-Saclay, DSM, DAPNIA, Service d'Astrophysique, 91191 Gif-sur-Yvette, France; paduc@cea.fr.

2 Institute of Astronomy, University of Cambridge, Madingley Road, Cambridge, CB3 0HA, UK.

${ }^{3}$ Departamento de Astronomía, Apdo. Postal 144, Guanajuato, Gto. 36000, Mexico.

${ }^{4}$ Max-Planck-Institut für Astrophysik, Karl-Schwarzschild Strasse 1, 85740 Garching bei München, Germany.

${ }^{5}$ Harvard Smithsonian Center for Astrophysics, MS 51, 60 Garden Street, Cambridge, MA 02138.

${ }^{6}$ Instituto de Astronomía, UNAM, Mexico.

${ }^{7}$ Universitätssternwarte, Geismarlandstr. 11, D-37083 Göttingen, Germany.

${ }^{8}$ CEA-Saclay, DSM, DAPNIA, Service d'Astrophysique, 91191 Gifsur-Yvette, France.

${ }^{9}$ IAFE, cc 67, snc 28 (1948), Buenos Aires, Argentina.
}

proved to be important tools to study the interaction, constraining the orbital parameters (Toomre \& Toomre 1972) of the collision. Recently, attempts have even been made to use the formation of tidal tails as a diagnostic of the mass distribution of halos within the framework of cold dark matter cosmologies (Dubinski, Mihos, \& Hernquist 1996; Springel \& White 1999). Much less attention has been paid to what goes on within these tidal features (see, for instance, Schombert, Wallin, \& Struck-Marcell 1990; Wallin 1990; Hibbard, Vacca, \& Yun 2000).

Detailed H I maps of a number of interacting systems (e.g., Hibbard \& van Gorkom 1996; Kaufman et al. 1997, and references therein) have shown that a large fraction of the gaseous component of colliding galaxies can be expelled into the galactic halos or even into the intergalactic medium as a result of tidal forces. In some systems, up to $90 \%$ of the atomic hydrogen is observed outside the optical disk (as in Arp 105, Duc et al. 1997). Even if part of this gas falls back toward its progenitors (Hibbard \& Mihos 1995), a significant amount of gas will be lost for the merger remnant for timescales of at least 1-10 Gyr. The stellar/gaseous tidal debris might be dispersed in the intergalactic/intracluster medium where the stellar component then contributes to the diffuse background light observed in clusters (Gregg \& West 1998) or recondense within the halo of the merger and form a new generation of galaxies: the so-called tidal dwarf galaxies (TDGs; see the review by Duc \& Mirabel 1999). 
TABLE 1

OBSERVING LoG

\begin{tabular}{|c|c|c|c|}
\hline Technique & Date & Telescope/Instrument & Technical Details \\
\hline \multirow[t]{3}{*}{ Optical imaging ............. } & $1992 \mathrm{Feb}$ & CFHT/PUMA & 1K SAIC1 CCD; 0 " $34^{\prime \prime}$ pixel $^{-1} ; B, V$ filters \\
\hline & $1995 \mathrm{Feb}$ & NTT/EMMI & 2K Tektronix CCD; $0^{\prime \prime} .27$ pixel $^{-1} ; B b, V, R$ filters (red arm) \\
\hline & 2000 Jan & ESO $3.6 \mathrm{~m} / \mathrm{EFOSC} 2$ & 2K Loral CCD; $0^{\prime \prime} 31$ pixel $^{-1}(2 \times 2$ binning $) ; B, V, R$ filters \\
\hline Near-infrared imaging ....... & $1996 \mathrm{Feb}$ & MPG-ESO $2.2 \mathrm{~m} / \mathrm{IRAC} 2 \mathrm{~B}$ & NICMOS3 IR array; $0^{\prime \prime} .5$ pixel $^{-1} ; J, H, K^{\prime}$ filters \\
\hline $\mathrm{H} \alpha$ imaging............... & $1995 \mathrm{Feb}$ & NTT/EMMI & 2K Tektronix CCD; 0 ".27 pixel $^{-1} ; \mathrm{HA} / 3, \mathrm{HA} / 0$ filters \\
\hline Long-slit spectroscopy ...... & $1995 \mathrm{Feb}$ & NTT/EMMI & 2K Tektronix CCD; $0^{\prime \prime} .27$ pixel $^{-1} 1$ 1".5 wide long slit, grism No. $3\left(360 \mathrm{~g} \mathrm{~mm}^{-1}\right)$ \\
\hline MOS spectroscopy.......... & 2000 Jan & ESO $3.6 \mathrm{~m} / \mathrm{EFOSC} 2$ & 1.7 wide slits; grism No. $11\left(300 \mathrm{~g} \mathrm{~mm}^{-1}\right)$ \\
\hline $\mathrm{H}$ I line map ................ & 1997 Sept & VLA/CS array & Details in Table 2 \\
\hline \multirow[t]{2}{*}{ CO line observations . } & 1999 Jun & IRAM $30 \mathrm{~m}$ & CO (1-0) beam: $22^{\prime \prime}$; details in Braine et al. (2000) \\
\hline & 1999 Nov & SEST & CO (1-0) beam: $44^{\prime \prime}$; details in Paper II \\
\hline
\end{tabular}

TDGs are typically found at the tip of tidal tails at distances between 20 and $100 \mathrm{kpc}$ from the merging objects, of which at least one should be a gas-rich galaxy. They are gas-rich objects that can be as massive as the Magellanic Clouds, form stars at a rate which might be as high as in blue compact dwarf galaxies (BCDGs) and seem dynamically independent from their parent galaxies. Although the observational evidence for the existence of recycled galaxies has been well established by now, their formation process is as yet not well understood. In particular it is not known when and under which conditions TDGs form during the interaction. This is one of the topics of the current paper.

Dating events is a general problem in extragalactic astronomy, which, however, may be more easily achieved for interacting systems via numerical simulations. The comparison of morphological and/or kinematical features with predictions based on numerical simulations provides strong indications on the age of the collision and therefore sets a constraint on the formation history of TDGs. Interacting galaxies observed just after the first perigalacticon are par- ticularly attractive. Whereas they have already developed long tails and bridges, their disks are still clearly separated and hence their intrinsic properties (i.e., orientation, sense, and amplitude of their rotation, which are key input parameters to the numerical models) can be well defined. In contrast, galaxies on course for their first interaction do not show strong perturbations before reaching perigalacticon and hence do not provide a simple handle on any timescale. On the other hand, evolved mergers have lost all memory of the initial properties.

Arp 245, an interacting system consisting of two spiral galaxies, NGC 2992 and NGC 2993, appears to be an interesting test case as it can be fairly easily modeled. Moreover, it is a relatively nearby system, at an adopted distance of 31 Mpc. ${ }^{10}$ Its prominent tidal tails hosts a tidal dwarf galaxy candidate, which because of its proximity can be studied in

\footnotetext{
${ }^{10}$ We use in this paper $H_{0}=75 \mathrm{~km} \mathrm{~s}^{-1} \mathrm{Mpc}^{-1}=100 h \mathrm{~km} \mathrm{~s}^{-1}$ $\mathrm{Mpc}^{-1}$. At the distance of Arp 245, 1' corresponds to $9 \mathrm{kpc}$.
}

TABLE 2

VLA OBSERVING PARAMETERS

\begin{tabular}{|c|c|}
\hline Parameter & Value \\
\hline Object & NGC 2992/93 (Arp 245) \\
\hline Instrument ...... & Very Large Array (VLA) \\
\hline Configuration & CS-array \\
\hline Observation date... & 1997 Sep 7 \\
\hline Total observing time ....... & $5.7 \mathrm{hr}$ \\
\hline Number of antennas .................. & 27 \\
\hline 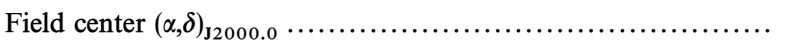 & $09^{\mathrm{h}} 45^{\mathrm{m}} 42^{\mathrm{s}},-14^{\circ} 19^{\prime} 35^{\prime \prime}$ \\
\hline 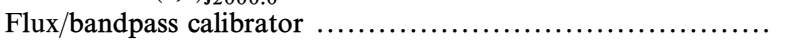 & $1331+305$ \\
\hline Phase calibrator........................ & $0902-142$ \\
\hline Central velocity $\left(V_{\text {heliocentric }}\right) \ldots .$. & $2341 \mathrm{~km} \mathrm{~s}^{-1}$ \\
\hline Observed baselines $(\min -\max ) \ldots$ & $0.05-3.4 \mathrm{~km}$ \\
\hline FWHP of primary beam .......... & $32^{\prime}$ \\
\hline System temperature........ & $35 \mathrm{~K}$ \\
\hline Correlator mode...$\ldots \ldots$ & 4 \\
\hline Total bandwidth per IF .... & $3.125 \mathrm{Mhz}\left(650 \mathrm{~km} \mathrm{~s}^{-1}\right)$ \\
\hline Number of channels per IF ......... & 32 \\
\hline Channel spacing & $21 \mathrm{~km} \mathrm{~s}^{-1}$ \\
\hline 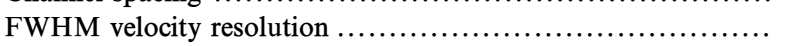 & $21 \mathrm{~km} \mathrm{~s}^{-1}$ \\
\hline rms noise per channel & $0.4 \mathrm{mJy}$ beam $^{-1}$ \\
\hline Smoothing applied ........................ & None, Gaussian \\
\hline 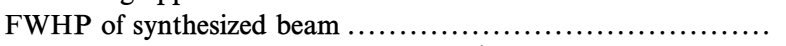 & $19^{\prime \prime} \times 14^{\prime \prime}, 35^{\prime \prime} \times 35^{\prime \prime}$ \\
\hline Conversion factor $T_{B}(\mathrm{~K}) / S\left(\mathrm{mJy}\right.$ beam $\left.^{-1}\right) \ldots \ldots \ldots \ldots \ldots \ldots \ldots \ldots$ & $2.18,0.495$ \\
\hline Conversion factor $N_{\mathrm{H}}\left(\mathrm{cm}^{-2}\right) / N_{\mathrm{H}}\left(\mathrm{mJy}\right.$ beam $\left.{ }^{-1} \mathrm{kms}^{-1}\right) \ldots \ldots$ & $3.97 \times 10^{18}, 9.02 \times 10^{17}$ \\
\hline
\end{tabular}




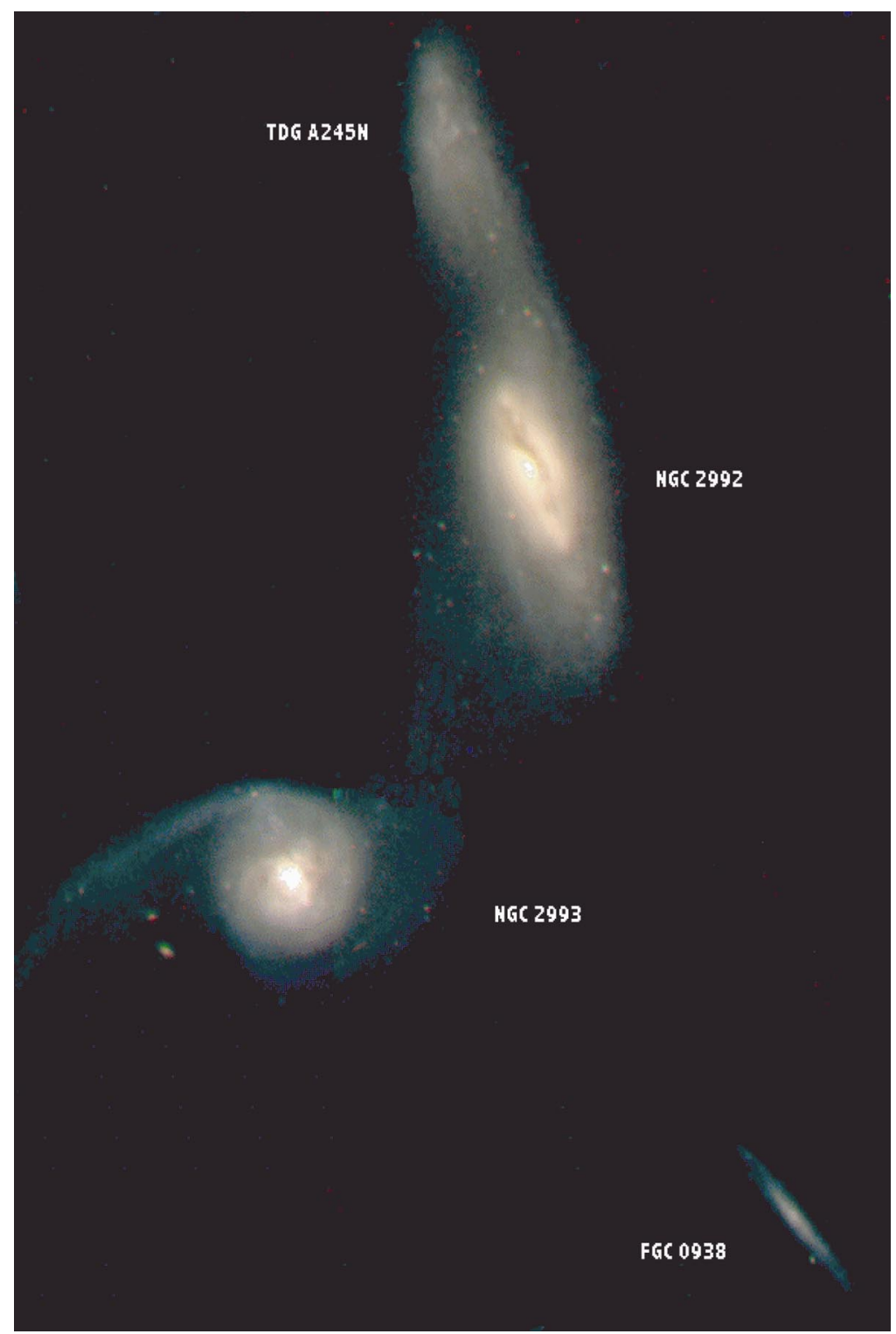

FIG. 1.-Optical true color image of Arp 245. The image is a combination of NTT + CFHT + ESO $3.6 \mathrm{~m} \mathrm{B-band} \mathrm{images}($ blue $)$, NTT + CFHT + ESO $3.6 \mathrm{~m} \mathrm{~V}$-band images (green) and NTT + ESO $3.6 \mathrm{~m} \mathrm{R}$-band images $($ red $)$. Bright foreground stars have been masked. The field of view is $6^{\prime} \times 8.2(54 \times 74$ $\mathrm{kpc}$ ). North is up, and east to the left. The system consists of two interacting spiral galaxies: NGC 2992 to the north and NGC 2993 to the southeast. The two galaxies, connected by a diffuse bridge, exhibit each a long stellar tidal tail. The one escaping from NGC 2992 hosts at its tip a star-forming tidal dwarf galaxy candidate, A245N. NGC 2993 appears bluer than NGC 2992 and is currently experiencing a vigorous starburst. A prominent dust lane in the latter galaxy lane partly obscures emission from the active Seyfert 1.9 nucleus. A blue edge-on dwarf galaxy, FGC 0938, is visible to the southeast.

detail. Finally, Arp 245 has already been well studied. NGC 2992 , in particular, has been the object of numerous articles focusing mostly on its active, Seyfert 1.9 nucleus. Radio continuum maps have revealed a striking pair of loops near the nucleus with a figure-eight shape (Ulvestad \& Wilson 1984) which has later on been detected at other wavelengths as well (Wehrle \& Morris 1988; Chapman et al. 2000). Glass (1997) has monitored the AGN in the near-infrared and has reported an outburst. Durret \& Bergeron (1987), Durret \& Bergeron (1988), Colbert et al. (1996), and Allen et al. (1999) have studied the ionization cone extending from the AGN and the surrounding extended emission-line regions. Evidence for outflow has been claimed by Colbert et al. (1996) and Márquez et al. (1998). NGC 2992 has been observed at many wavelengths from the X-ray regime, where it is a strong emitter (Marshall, Warwick, \& Pounds 1981; Gilli et al. 2000), to the centimeter wavelengths (Condon et al. 1982). Single-dish H I (Mirabel \& Wilson 1984) and CO (Sanders \& Mirabel 1985) data are also available. NGC 2993 is mentioned in several catalogs of starburst galaxies but has not yet been the subject of any individual study. Surprisingly, the fact that both galaxies are partaking in a spectacular interaction has largely been ignored, apart from the photometric work by Schombert et al. (1990).

We decided to subject Arp 245 to a comprehensive multiwavelength study. In this first paper, we detail the observations, present in particular the first complete $\mathrm{H}$ I map of the system, and assess the status of the interaction with the help of numerical $N$-body/hydrodynamical simulations. We then focus on the tidal features, emphasizing the properties of the tidal dwarf galaxy candidate. Based on these results, and of similar such systems from the literature, we attempt 


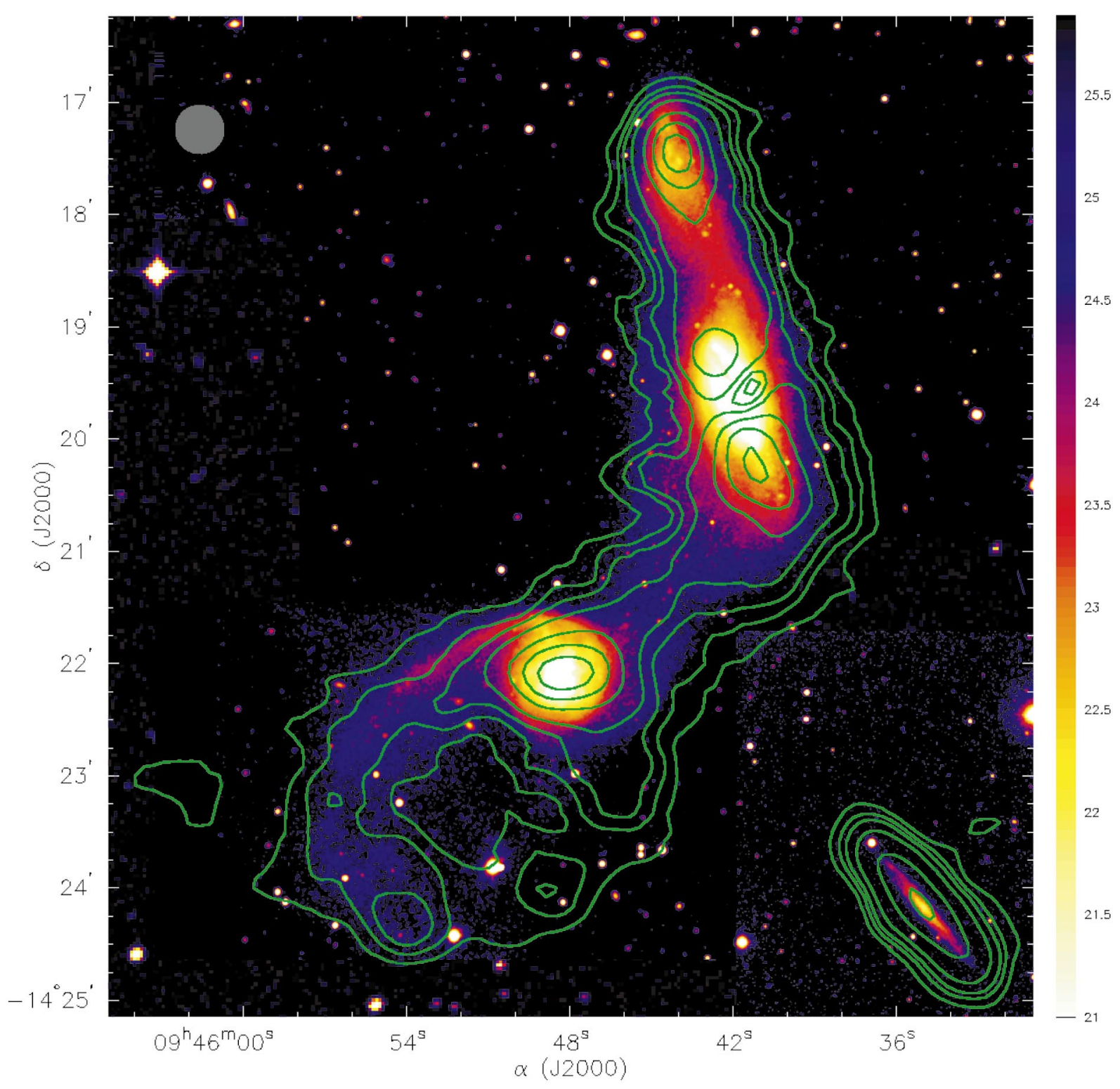

Fig. 2.- - H I distribution in Arp 245. The VLA H I contours are superposed on a $V$-band image of the system. The contour levels are $1,2,3,5,10,15$, and $20 \times 10^{20} \mathrm{~cm}^{-2}$. The radio beam is indicated at the top left of the image. The optical background picture consists of recombined calibrated $V$-band images from different telescopes displayed with an intensity scale in magnitudes per square arcsecond, as indicated to the right. Although there is an overall agreement between the distribution of the stellar and gaseous components, some differences can be noticed between the two interacting galaxies. Toward NGC 2992, the H I shows a peak at the location of the tidal dwarf candidate. H I is seen in absorption against the radio continuum from the nucleus. Toward NGC 2993, the H I map has a ringlike structure. Its western part has no optical counterpart. A detached cloud can be seen east of the ring. The VLA observations revealed at the same distance as Arp 245 a gas-rich edge-on dwarf galaxy, FGC 0938, which is visible to the southwest. This object, which looks remarkably unperturbed, might be falling toward the NGC 2992/93 system.

to better understand the formation process of TDGs. In a second paper (Paper II), we will concentrate on the internal properties of NGC 2992, and study in particular its active galactic nucleus (AGN) and the ionization filaments.

\section{OBSERVATIONS}

Tables 1 and 2 summarize our multiwavelength observations ${ }^{11}$ of Arp 245 and lists technical details. In the next sections we will discuss these observations in turn.

${ }^{11}$ Partly carried out at the European Southern Observatory, La Silla, Chile (ESO No 54.A-0606, 56.A-0757, 64.N-0163 and 64.N-0361).

\subsection{Optical and Near-Infrared Broadband Imaging}

Optical broadband $B V R$ images of Arp 245 have been collected in 1995 February with the $3.5 \mathrm{~m}$ NTT at la Silla observatory. The red arm of EMMI has been used. The weather conditions were photometric and the seeing varied between $0^{\prime \prime} \cdot 9$ and $1^{\prime \prime} .2$. The $9.2 \times 8.7$ field of view of the $R$-band image covered the entire interacting system including the tidal features whereas the $B^{12}$ - and $V$-band images were somewhat offset and missed a small part of the southern tail. However, $B$ and $V$ images of this part of the

\footnotetext{
${ }^{12}$ The $B$-like filter, $B b$, optimized for the red arm of EMMI, has actually been used.
} 
TABLE 3

Properties OF THE INTERACTING PARTNERS

\begin{tabular}{|c|c|c|c|}
\hline Parameter & NGC 2992 & NGC 2993 & FGC 0938 \\
\hline$\alpha(\mathrm{J} 2000.0)$. & $09^{\mathrm{h}} 45^{\mathrm{m}} 42^{\mathrm{s}} .0$ & $09^{\mathrm{h}} 45^{\mathrm{m}} 48^{\mathrm{s}} 3$ & $09^{\mathrm{h}} 45^{\mathrm{m}} 34^{\mathrm{s}} .4$ \\
\hline$\delta(\mathrm{J} 2000.0) \ldots$ & $-14^{\circ} 19^{\prime} 35^{\prime \prime}$ & $-14^{\circ} 22^{\prime} 05^{\prime \prime}$ & $-14^{\circ} 24^{\prime} 16^{\prime \prime}$ \\
\hline Heliocentric systemic velocity $\left(\mathrm{km} \mathrm{s}^{-1}\right) \ldots \ldots$ & 2330 & 2420 & 2500 \\
\hline 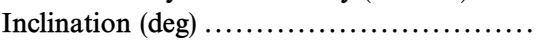 & 70 & 20 & 80 \\
\hline Morphological type...$\ldots \ldots \ldots \ldots \ldots \ldots \ldots$ & Sa pec & Sab & Scd \\
\hline Corrected blue total magnitude .............. & 12.23 & 12.51 & 16.95 \\
\hline Absolute blue magnitude ..................... & -20.23 & -19.95 & -15.51 \\
\hline Blue luminosity $\left(10^{10} L_{\odot}\right) \ldots \ldots \ldots \ldots \ldots \ldots$ & 1.92 & 1.49 & 0.02 \\
\hline FIR luminosity $\left(10^{10} L_{\odot}\right) \ldots \ldots \ldots \ldots \ldots \ldots$ & 1.80 & 2.54 & $\ldots$ \\
\hline 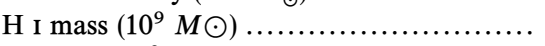 & 1.97 & 1.05 & 0.70 \\
\hline 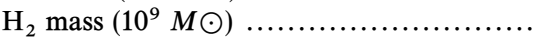 & 1.19 & 0.39 & $\ldots$ \\
\hline $\mathrm{H} \alpha+[\mathrm{N} \mathrm{II}]$ luminosity $\left(10^{40} \mathrm{ergs} \mathrm{s}^{-1}\right) \ldots \ldots$ & 17 & 28 & $\ldots$ \\
\hline $20 \mathrm{~cm}$ emission $(\mathrm{mJy}) \ldots \ldots \ldots \ldots \ldots \ldots \ldots \ldots$ & 180 & 45 & $\ldots$ \\
\hline$B-V$ color index (total, outskirts) $\ldots$. & $0.79,0.62$ & $0.33,0.40$ & $0.46, \ldots$ \\
\hline$V-R$ color index (total, outskirts) .......... & $0.51,0.43$ & $0.40,0.39$ & $0.30, \ldots$ \\
\hline$V-K^{\prime}$ color index (total, outskirts) $\ldots \ldots \ldots$ & $3.60,3.03$ & $\ldots, \ldots$ & $\ldots, \ldots$ \\
\hline$J-H$ color index (total, outskirts) $\ldots \ldots \ldots \ldots$ & $0.64,0.41$ & $\ldots, \ldots$ & $\ldots, \ldots$ \\
\hline$H-K^{\prime}$ color index (total, outskirts) $\ldots \ldots \ldots \ldots$ & $0.46,0.47$ & $\ldots, \ldots$ & $\ldots, \ldots$ \\
\hline
\end{tabular}

NoTE.-Sources: NED (positions, FIR fluxes), LEDA (morphology, corrected blue magnitudes). The other data are from this paper. The velocity of FGC 0938 is the H I velocity. All optical magnitudes have been corrected for Galactic extinction. The magnitudes labeled "outskirts" have been measured with a polygonal aperture that encompassed the outermost regions of NGC 2992/ 93 , at a minimum radial distance of $30^{\prime \prime}$ from the nucleus and avoiding the prominent dust lane.

system had been previously taken with the PUMA camera on the Canada-France-Hawaii Telescope (CFHT) ${ }^{13}$ in 1992 February. The weather conditions at CFHT were poor and the seeing was $1^{\prime \prime}$. Finally, $B V R$ images of a field situated to the southwest of NGC 2993 were obtained in 2000 January with the ESO $3.6 \mathrm{~m}$ telescope. The weather conditions were photometric and the seeing was $1^{\prime \prime}$. Images from the three telescopes were eventually combined. Landolt fields of photometric standard stars (Landolt 1992) were observed for flux calibration.

Near-infrared $J H K^{\prime}$ images of NGC 2992 were obtained in 1996 February with the IRAC2B camera installed on the MPG/ESO $2.2 \mathrm{~m}$. The field of view of each individual image was $2^{\prime} \cdot 1 \times 2{ }^{\prime} \cdot 1$. Sky images were taken of adjacent fields offset by $2^{\prime}$. The entire field of NGC 2992, including the tidal tail, was covered with a mosaic technique. The highest effective integration time was reached toward the tail which was observed for a total of 15 minutes in $J, 12$ minutes in $H$, and 13 minutes in $K^{\prime}$. Several photometric standard stars from the list of UKIRT faint IR standards (Casali \& Hawarden

${ }^{13}$ The CFHT is operated by the National Research Council of Canada, the Centre National de la Recherche Scientifique de France and the University of Hawaii.
1992) were observed under photometric conditions. The seeing varied between 1 .". 3 and 1.5 .

The reduction of the optical/NIR data has been performed within IRAF with standard tasks from the CCDRED package complemented by self-written scripts which perform a semiautomatic processing of the NIR data set. An astrometric correction achieving a precision of $\sim 0.3$ was performed to each optical/NIR image using the positions of several tens of stars from the USNO A1.0 astrometric catalog (Monet 1996) queried via the ESO SKYCAT browser. The frames were corrected for distortions during this process. They were registered, PSF-matched and combined to produce color maps. An optical "true-color" image of the system was constructed from the combination of the NTT/CFHT/3.6 $\mathrm{m} B, V$, and $R$ images. It is shown in Figure 1. The monochromatic $V$-band image is displayed in Figure 2.

Photometry was carried out on the registered images with the DIGIPHOT package in IRAF. Polygonal apertures were chosen to enclose respectively the disks of NGC 2992 and NGC 2993, their outer regions only, the bridge between both systems, the tidal tails, and the tidal dwarf galaxy. Because of the complex geometry of the system, the sky level was measured manually and averaged at numerous positions surrounding the objects. This procedure was

TABLE 4

Properties of the Tidal Features

\begin{tabular}{|c|c|c|c|}
\hline Parameter & NGC 2992 Tail & NGC 2993 Tail & Bridge \\
\hline Optical extent $(\mathrm{kpc})$. & 15.5 & 26.6 & 14.4 \\
\hline$B$ magnitude ${ }^{a}$. & $14.63 \pm 0.01$ & $15.22 \pm 0.01$ & $15.51 \pm 0.1$ \\
\hline$B-V^{\mathrm{a}}$. & $0.57 \pm 0.02$ & $0.39 \pm 0.02$ & $0.50: \pm 0.2$ \\
\hline$V-R^{\mathrm{a}}$. & $0.42 \pm 0.02$ & $0.45 \pm 0.02$ & $0.45: \pm 0.2$ \\
\hline H I mass $\left(10^{8} M \odot\right)$ & 11.6 & 8.5 & 3.7 \\
\hline Peak H I column densities $\left(10^{21} \mathrm{~cm}^{-2}\right)$. & 2.3 & 0.4 & 0.65 \\
\hline$V$-band max surface brightness $\left(\mathrm{mag} \operatorname{arcsec}^{-2}\right) \ldots \ldots$ & 22.1 & 23.3 & 24.0 \\
\hline
\end{tabular}

${ }^{a}$ Corrected for Galactic extinction. 


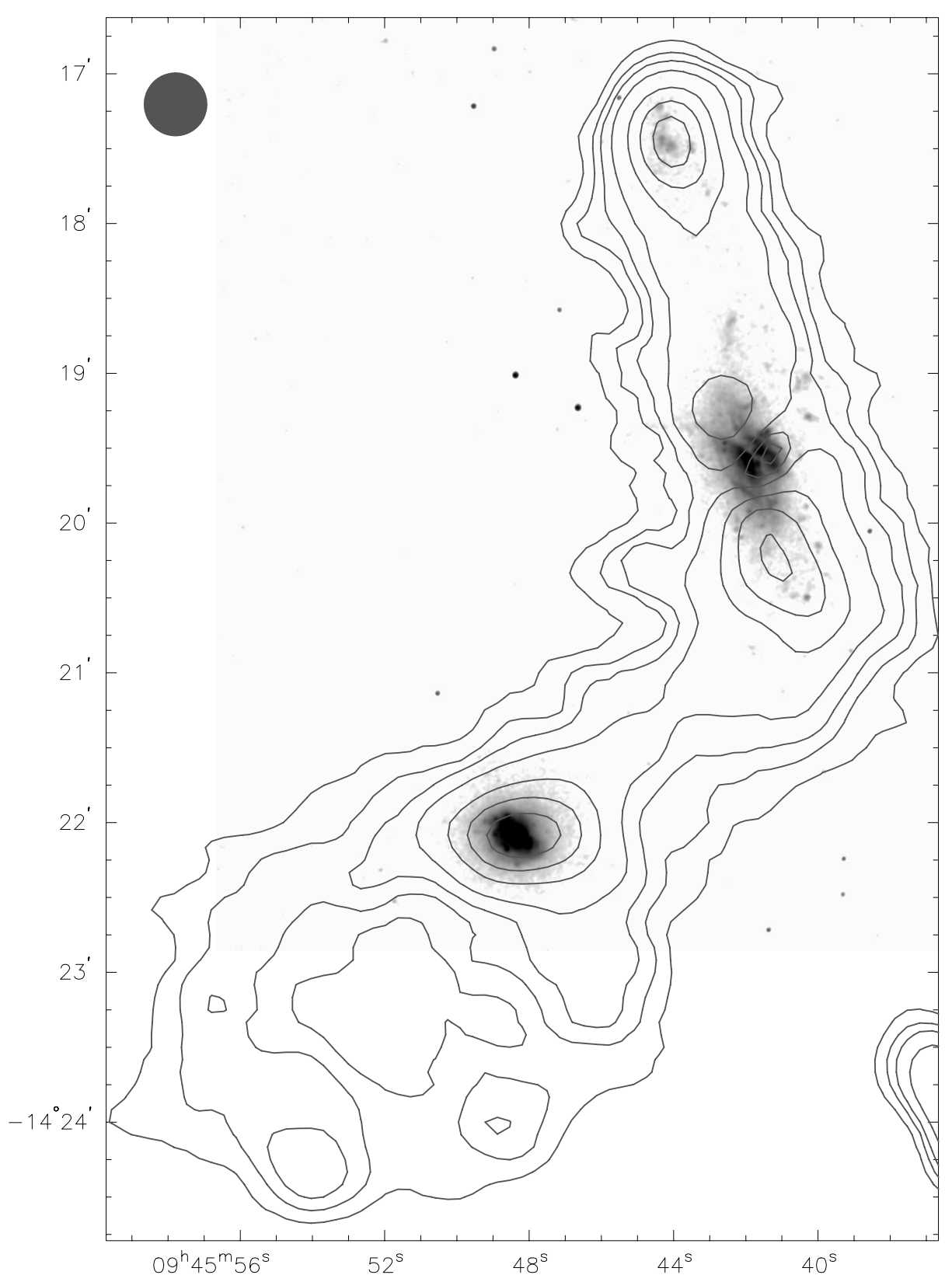

FIG. 3.-NTT H $\alpha+[\mathrm{N}$ II $]$ image of Arp 245. The H I map with the same contours as in Fig. 2 is superposed. The colored background indicates the field of view covered by the narrowband image. The $\mathrm{H} \alpha$ image is displayed on a logarithmic intensity scale. The sensitivity limit is $0.4 \times 10^{-16} \mathrm{ergs}^{-1} \mathrm{~cm}^{-2}$ $\operatorname{arcsec}^{-2}$. Toward NGC 2993 and A245N, H II regions are concentrated within H I clumps and trace star-forming regions. The biconical structure of the H $\alpha$ emission in the inner regions of NGC 2992 and extending to larger scales in the presence of numerous ionization filaments betray strong nuclear activity in this Seyfert-1.9 galaxy.

chosen so as to ensure that sky contamination by stars or galaxies would be minimal. The photometric accuracies take into account variations in the sky background as well as photon noise. The magnitudes, corrected for Galactic extinction are listed in Tables 3, 4, and 5. Circular aperture photometry of both NGC 2992 and NGC 2993 was performed as well, for comparison purposes. In the optical, our $V$ and $R$ magnitudes differ by less than 0.05 mag with data in the literature (Prugniel \& Heraudeau 1998) whereas in the $B$ band, the agreement is not as good. The $B$ magnitudes were systematically too faint by $0.1 \mathrm{mag}$. A scatter in the color terms in the blue is expected since the EMMI $B b$ filter which we have used slightly differs from a Bessel $B$ filter. As the system does not show large variations in color, we have applied to all $B$-band magnitudes a correction corresponding to an offset of $0.1 \mathrm{mag}$. This post-calibration has been later on validated using the $B$-band photometric measurements obtained in 2000 January. In the near-infrared, comparisons are more difficult because of the intrinsic variability of the nuclear flux. Glass (1997) monitored the AGN and measured excursions of the central $K$-band flux of up to $0.6 \mathrm{mag}$ due to outbursts. Colors appear to be less affected though and our $J-H$ and $H-K^{\prime}$ colors are within $0.1 \mathrm{mag}$ of those of Alonso-Herrero et al. (1998). On average our optical/NIR photometry might be affected by systematic errors of up to $0.1 \mathrm{mag}$. Note that the instrumental errors quoted in Tables 4 and 5 do not take this into account. 


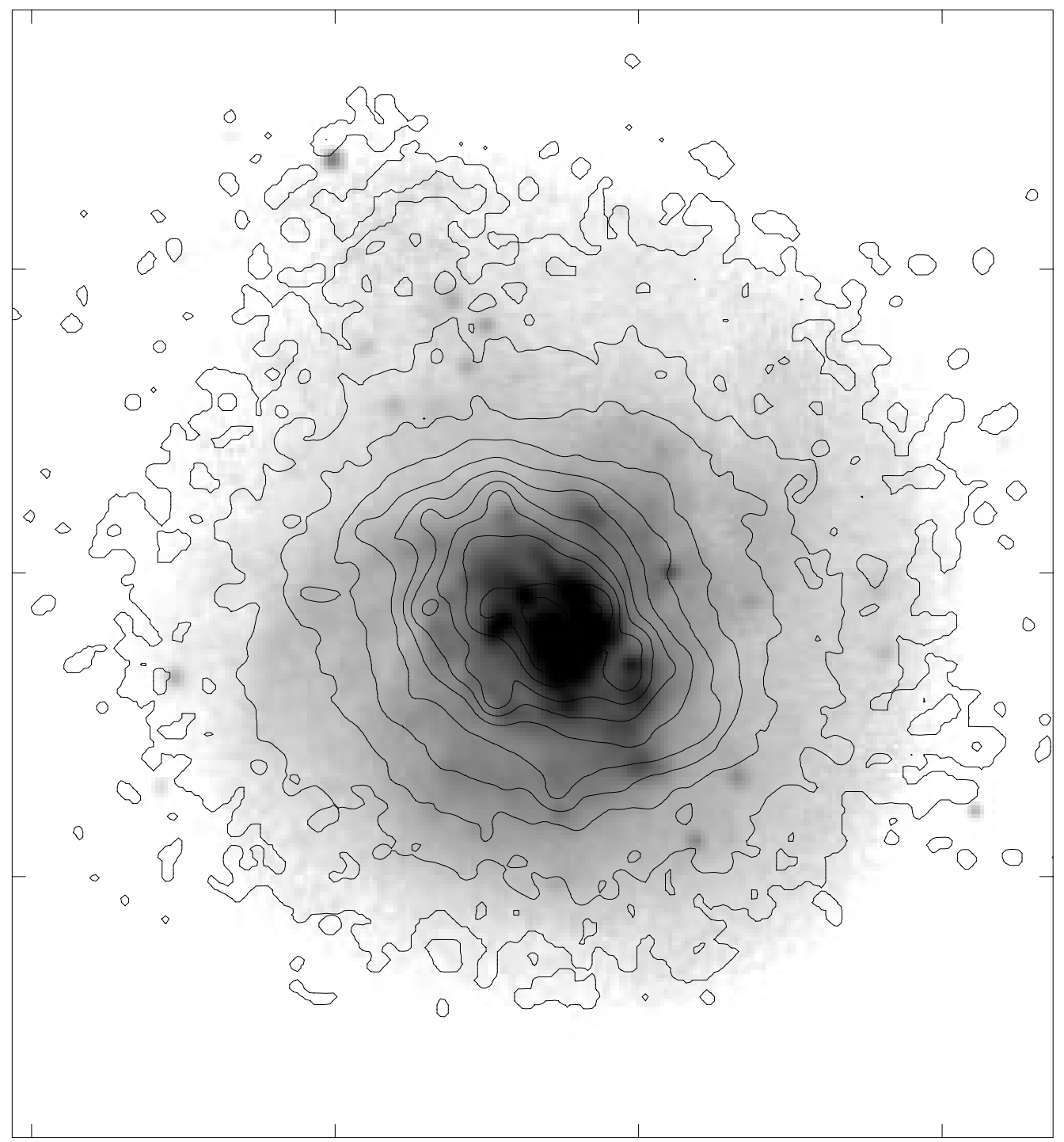

Fig. 4.-Calibrated $R$-band image of NGC 2993. H $\alpha$ contours are superposed. Levels differ by a multiplicative factor of 2 . The lowest contour is $0.4 \times 10^{-16} \mathrm{ergs} \mathrm{s}^{-1} \mathrm{~cm}^{-2} \operatorname{arcsec}^{-2}$. The interval between the tick marks is $20^{\prime \prime}$. North is up, and east to the left.

\section{2. $\mathrm{H} \alpha$ Imaging}

An image with a narrowband filter centered on the redshifted $\mathrm{H} \alpha$ line has been obtained with EMMI during the NTT run. The filter which has a width (FWHM) of $66 \AA$ includes light from $\mathrm{H} \alpha$ and $[\mathrm{N}$ II]. The image covers all of NGC 2992, including its tidal plume and bridge, and the disk of NGC 2993 with part of its tidal tail. Only the southern most region has been missed. The exposure time was 15 minutes. The continuum was obtained by using a similar narrowband filter but offset in wavelength. A halftone $\mathrm{H} \alpha$ image is displayed in Figure 3 and intensity contours of the line emission toward NGC 2993 and toward the northern tidal tail are shown in Figure 4 and Figure 16, respectively. A contour map toward NGC 2992 will be presented in Paper II. Our $\mathrm{H} \alpha$ image shows a number of structures not seen in the maps previously published (see, e.g., Colbert et al. 1996; Allen et al. 1999 for some recent ones), such as long ionization filaments outside the disk of NGC 2992 and $\mathrm{H}$ II regions at the tip of the northern tidal plume. Flux calibration was achieved by observing a spectroscopic standard star from the list of Hamuy et al. (1992) observed with same setup. Our integrated flux toward NGC 2992 agrees to within $20 \%$ with the flux measured by Colbert et al. (1996).

\subsection{Optical Spectroscopy}

Long-slit spectroscopic observations were carried out in 1995 February with the NTT and EMMI. The grism used has an intrinsic resolution of $9 \AA$. Coupled with the red-arm CCD, it covers the wavelength range $3800-8400 \AA$. Two spectra were obtained along an axis roughly parallel to the morphological axis of NGC 2992. The position angles were $-18^{\circ}$ and -20.2 . The $1^{\prime \prime} 5$ wide slits encompassed the western regions of NGC 2992 and two of the brightest clumps of the tidal dwarf galaxy at the tip of the plume. The total integration time was 45 minutes divided in three exposures. The spectra of several spectroscopic standard stars from the list of Hamuy et al. (1992) were measured through a wide slit. Weather conditions were photometric. A spectroscopic follow-up was performed in 2000 January with the EFOSC2 instrument installed on the ESO $3.6 \mathrm{~m}$ telescope. The MOS mode offered by punched masks was used 
TABLE 5

Properties of the Tidal ObJect A245N

\begin{tabular}{|c|c|}
\hline Parameter & Value \\
\hline$\alpha(\mathrm{J} 2000.0) \ldots \ldots$ & $09^{\mathrm{h}} 45^{\mathrm{m}} 44^{\mathrm{s}} .2$ \\
\hline 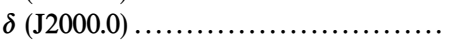 & $-14^{\circ} 17^{\prime} 29^{\prime \prime} .1$ \\
\hline Heliocentric $\mathrm{H}$ I velocity .............. & $2175 \mathrm{~km} \mathrm{~s}^{-1}$ \\
\hline Mean optical velocity ................ & $2240 \mathrm{~km} \mathrm{~s}^{-1}$ \\
\hline Distance to progenitor................ & $19.2 \mathrm{kpc}$ \\
\hline Size $\left(D_{24.5}\right) \ldots \ldots \ldots \ldots \ldots \ldots \ldots \ldots \ldots \ldots$ & $5.0 \times 10.5 \mathrm{kpc}$ \\
\hline Blue magnitude $^{a} \ldots \ldots \ldots \ldots \ldots \ldots \ldots$ & $15.21 \pm 0.01$ \\
\hline Absolute blue magnitude ${ }^{a}$.. & $-17.25 \pm 0.01$ \\
\hline Blue luminosity ${ }^{\mathrm{a}}$............ & $12.3 \times 10^{8} L_{\odot}$ \\
\hline Blue central surface brightness ${ }^{\mathrm{a}} \ldots \ldots$. & $22.4 \mathrm{mag} \operatorname{arcsec}^{-2}$ \\
\hline \multirow[t]{3}{*}{ Optical/NIR colors ${ }^{\mathrm{a}} \ldots \ldots \ldots \ldots \ldots \ldots$} & $B-V=0.55 \pm 0.01$ \\
\hline & $V-R=0.41 \pm 0.02, V-K^{\prime}=3.14 \pm 0.02$ \\
\hline & $J-H=0.32 \pm 0.03, H-\mathrm{K}^{\prime}=0.51 \pm 0.03$ \\
\hline 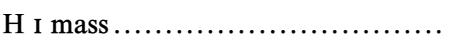 & $8.9 \times 10^{8} M \odot$ \\
\hline 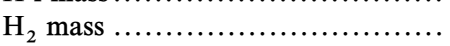 & $1.4 \times 10^{8} M \odot$ \\
\hline $\mathrm{H} \alpha+[\mathrm{N}$ II $]$ luminosity .............. & $0.74 \times 10^{40} \mathrm{ergs} \mathrm{s}^{-1}$ \\
\hline Star formation rate ${ }^{b} \ldots \ldots \ldots \ldots \ldots \ldots$ & $0.03(0.13) M \odot \mathrm{yr}^{-1}$ \\
\hline Intrinsic extinction ${ }^{c} \ldots$ & $A_{B}=2.6 \mathrm{mag}$ \\
\hline
\end{tabular}

${ }^{\text {a }}$ Corrected for Galactic extinction.

${ }^{b}$ Corrected for Galactic and total extinction (values in parentheses).

${ }^{c}$ Derived from the Balmer decrement toward TDG 4.

to obtain the optical spectra of several extended objects near NGC 2992 as well as some new condensations in its tidal dwarf. The grism used had a wider wavelength coverage but a slightly lower spectral resolution. Five exposures were obtained and the total integration time was 100 minutes.

Data reduction, wavelength, and flux calibrations were performed with the TWODSPEC package within IRAF following standard procedures. Line fluxes and errors were measured with the IRAF SPLOT task. Data were corrected for extinction using the formula

$$
\left[\frac{I(\lambda)}{I(\mathrm{H} \beta)}=\frac{F(\lambda)}{F(\mathrm{H} \beta)} \times 10^{c \times f(\lambda)}\right]
$$

where $F(\lambda)$ is the observed line flux, $f(\lambda)$ the reddening function taken from Torres-Peimbert, Peimbert, \& Fierro (1989), and $c$ the logarithmic reddening correction at $\mathrm{H} \beta$ obtained from a constant $\mathrm{H} \alpha / \mathrm{H} \beta$ Balmer decrement of 2.85 . The lines were not corrected for underlying stellar absorption. Table 6 displays the values of $c$, the absolute flux and equivalent width of the $\mathrm{H} \beta$ line and the observed/corrected fluxes relative to $\mathrm{H} \beta$ of the principal lines. The accuracy of the absolute flux calibration has been checked using our $\mathrm{H} \alpha$ narrowband image. The integrated $\mathrm{H} \alpha$ fluxes of several emission-line regions extracted from the $\mathrm{H} \alpha$ image and from the slit spectra agree to within 5\% to $15 \%$.

\subsection{VLA H I Observations}

We obtained observations with the Very Large Array $(\mathrm{VLA})^{14}$ of Arp 245 in the $21 \mathrm{~cm}$ line of neutral hydrogen

\footnotetext{
${ }^{14}$ The VLA is operated by the National Radio Astronomy Observatory, a facility of the National Science Foundation operated under cooperative agreement by Associated Universities for Research in Astronomy, Inc.
}

(H I) in September 1997, using the CS-array. ${ }^{15}$ We used the calibrator $1331+305$ for absolute flux calibration and 0902142 as secondary calibrator. We determined a flux density for 0902-142 of 2.89 $\pm 0.01 \mathrm{Jy}$. The data were taken in correlator mode 4 with two passbands (or IFs) measuring both right and left hand polarization. Each band contained 32 channels, and we used an eight-channel overlap between the bands. The first and last three channels of each band were discarded because of the decreasing gain toward the edges of the bandpass. Full details of the observations are presented in Table 2.

Data reduction and calibration were performed using the Astronomical Image Processing System (AIPS) package, following standard procedures. The uv data from each of the two IFs were calibrated separately. The visibilities were inspected and bad data points due to interference were removed. Because of the presence of strong solar interference at the lowest spatial frequencies, we excluded baselines shorter than $1 \mathrm{k} \lambda$ (or about $210 \mathrm{~m}$ ) in the calibration. After the standard amplitude and phase calibration we went through one cycle of self-calibration (phase only), improving the final quality of the images. As reference source we used the bright nuclear emission of NGC 2992 which has a flux density of $190 \pm 2 \mathrm{mJy}$.

For each IF we generated "dirty" data cubes of line plus continuum emission. After running several tests we decided that the best maps were those made with the task IMAGR using the default values for the ROBUST weighting scheme and including the entire observed uv range. The first 15 and the last 15 channels of IF1 and IF2 respectively, were found to be free of line emission. From each corresponding IF the

\footnotetext{
${ }^{15}$ This configuration results in a resolution equivalent to that of $\mathrm{C}$ configuration, but has some antennae placed at locations usually employed for D configuration, with the aim of providing sufficient short spacing information so that separate D-configuration observations are not necessary.
} 


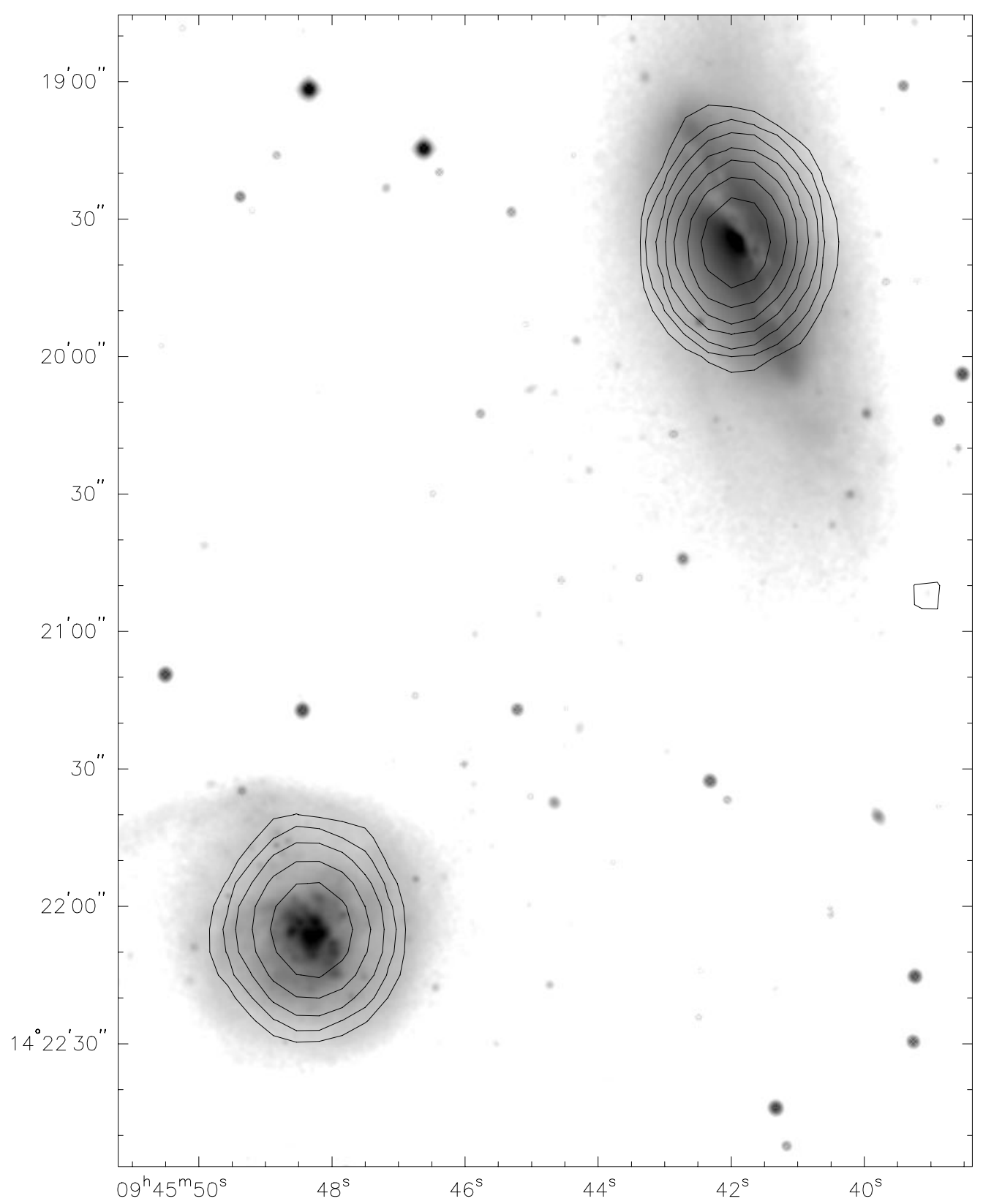

FIG. 5. $-20 \mathrm{~cm}$ radio continuum map superposed on the $R$-band image of Arp 245. Levels are 1.8, 3.5, 6.8, 13.2, 25.7, 50.1, 97.3, and 189 mJy beam ${ }^{-1}$.

TABLE 6

SPECTROPHOTOMETRY OF A245N

\begin{tabular}{|c|c|c|c|}
\hline Parameter & TDG $1^{\mathrm{a}}$ & TDG $3^{\mathrm{a}}$ & TDG $4^{\mathrm{a}}$ \\
\hline [O II] 3727 & & $183 \pm 23,401 \pm 50$ & \\
\hline $\mathrm{H} \beta 4861.3 \ldots \ldots \ldots \ldots \ldots$ & $100 \pm 37,100 \pm 37$ & $100 \pm 18,100 \pm 18$ & $100 \pm 17,100 \pm 17$ \\
\hline [O III] 5006.9 . & $35 \pm 28,32 \pm 26$ & $74 \pm 8,68 \pm 7$ & $88 \pm 17,82 \pm 15$ \\
\hline$[\mathrm{N} \mathrm{III}] 6548.1 \ldots$ & $125 \pm 61,51 \pm 25$ & $\ldots, \ldots$ & $80 \pm 18,37 \pm 8$ \\
\hline $\mathrm{H} \alpha 6562.8 \ldots$. & $705 \pm 172,285 \pm 70$ & $785 \pm 80,285 \pm 29$ & $622 \pm 64,285 \pm 29$ \\
\hline$[\mathrm{N} \mathrm{II}] 6583.4 \ldots .$. & $338 \pm 93,135 \pm 37$ & $290 \pm 33,104 \pm 12$ & $291 \pm 33,132 \pm 15$ \\
\hline$[$ S II $] 6716.4 \ldots \ldots \ldots \ldots \ldots \ldots \ldots \ldots \ldots \ldots \ldots$ & $200 \pm 68,74 \pm 25$ & $\ldots, \ldots$ & $143 \pm 25,60 \pm 11$ \\
\hline$[\mathrm{S}$ II $] 6730.8 \ldots \ldots \ldots \ldots \ldots \ldots \ldots \ldots \ldots \ldots \ldots \ldots \ldots$ & $101 \pm 41,37 \pm 15$ & $\ldots, \ldots$ & $79 \pm 16,33 \pm 7$ \\
\hline Reddening correction factor $(c) \ldots \ldots$ & 1.17: & 1.31 & 1.01 \\
\hline $\mathrm{H} \beta$ equivalent width $(\AA)$ & $6 \pm 2$ & $9 \pm 2$ & $13 \pm 2$ \\
\hline
\end{tabular}

${ }^{a}$ First values correspond to the observed fluxes relative to $\mathrm{H} \beta=100$; second values correspond to the extinction-corrected relative intensities. 


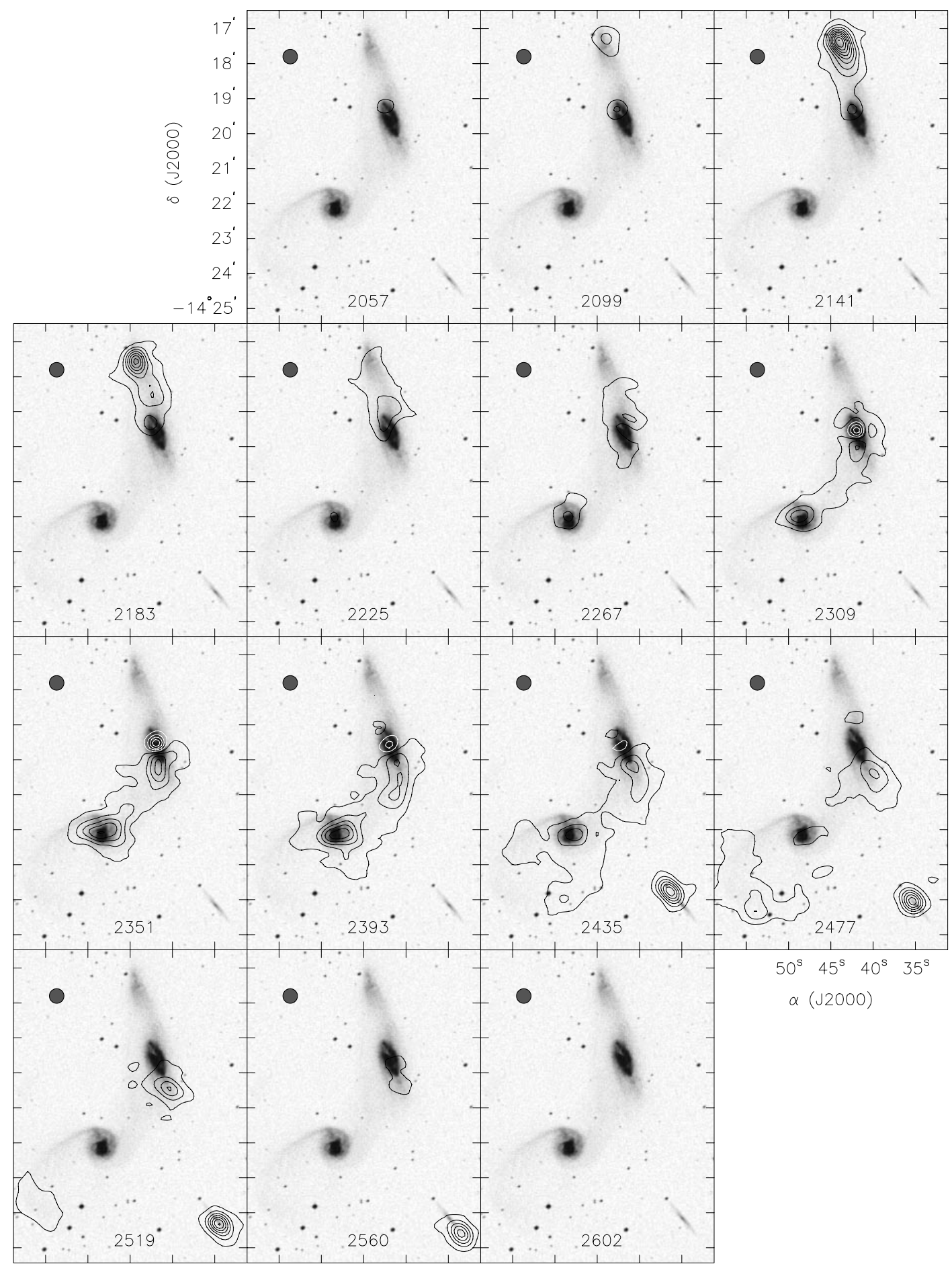

FIG. 6.-H I channel maps of Arp 245. The contours in each channel are superposed on an optical DSS image of the system. Black contours correspond to $\mathrm{H}$ I seen in emission and white contours $\mathrm{H}$ I seen in absorption. The lowest level is $\pm 0.8 \mathrm{mJy} \mathrm{beam}^{-1}$ and the interval is $1.8 \mathrm{mJy} \mathrm{beam}^{-1}$. The velocity of each channel in $\mathrm{km} \mathrm{s}^{-1}$ is indicated at the bottom of each frame. The VLA beam size is indicated in the upper left-hand corner. 
average of those line-free channels was subtracted to construct data cubes with line emission only. An average of all line-free channels from both IFs was used to construct a map of the continuum emission. This map, after cleaning, is shown in Figure 5. Besides the nuclear emission due to NGC 2992 we see emission corresponding to the nucleus of NGC 2993. This emission is resolved and measures 12 ".2 $\times 9{ }^{\prime \prime} 4$ at a position angle of $58^{\circ}$. Its peak flux corresponds to $49 \mathrm{mJy}$.

After continuum subtraction the maps were inspected and cleaned, and the two IFs were appropriately merged. In order to isolate the $\mathrm{H} \mathrm{I}$ emission in the maps from the noise, the cube, which has originally a resolution of 19 ". $4 \times 14$ ".4 was convolved to a circular beam of $35^{\prime \prime}$. We clipped this cube at a level of twice the rms noise. We inspected all features in this cube, retaining only those regions which show emission in at least five consecutive channels. We then went back to the original, high-resolution cube and applied conditional blanking, retaining emission in the highresolution cube from only those regions which were pre- served in the processed $35^{\prime \prime}$ cube. As a fringe benefit this process removed most if not all traces of solar interference. Besides emission, $\mathrm{H} \mathrm{I}$ absorption is seen toward the strong nuclear source in NGC 2992. We ensured that this was preserved in the conditional blanking process. In addition to a blanked cube at the highest resolution $\left(199^{\prime \prime} 4 \times 14^{\prime \prime} 4\right)$ we produced lower resolution cubes as well, such as at $25^{\prime \prime} \times 25^{\prime \prime}$ and $30^{\prime \prime} \times 30^{\prime \prime}$. As a last step we obtained the moments of the cubes. The conversion factors from millijanskys per beam to Kelvin brightness temperatures are listed in Table 2.

Figure 6 displays a mosaic of the $\mathrm{H}$ i channel maps at a resolution of 25", showing every second channel, superposed on an image obtained from the DSS of the same area. The final $\mathrm{H}$ I cube consists of 32 channels with velocities ranging from $2655 \mathrm{~km} \mathrm{~s}^{-1}$ to $2006 \mathrm{~km} \mathrm{~s}^{-1}$ with a channel spacing of $21 \mathrm{~km} \mathrm{~s}^{-1}$. Emission is found from $2592 \mathrm{~km} \mathrm{~s}^{-1}$ to $2048 \mathrm{~km} \mathrm{~s}^{-1}$. Starting at $2267 \mathrm{~km} \mathrm{~s}^{-1}$ and extending over some $200 \mathrm{~km} \mathrm{~s}^{-1}$ to $2477 \mathrm{~km} \mathrm{~s}^{-1}$ we see strong absorption against the nuclear continuum source of NGC 2992.

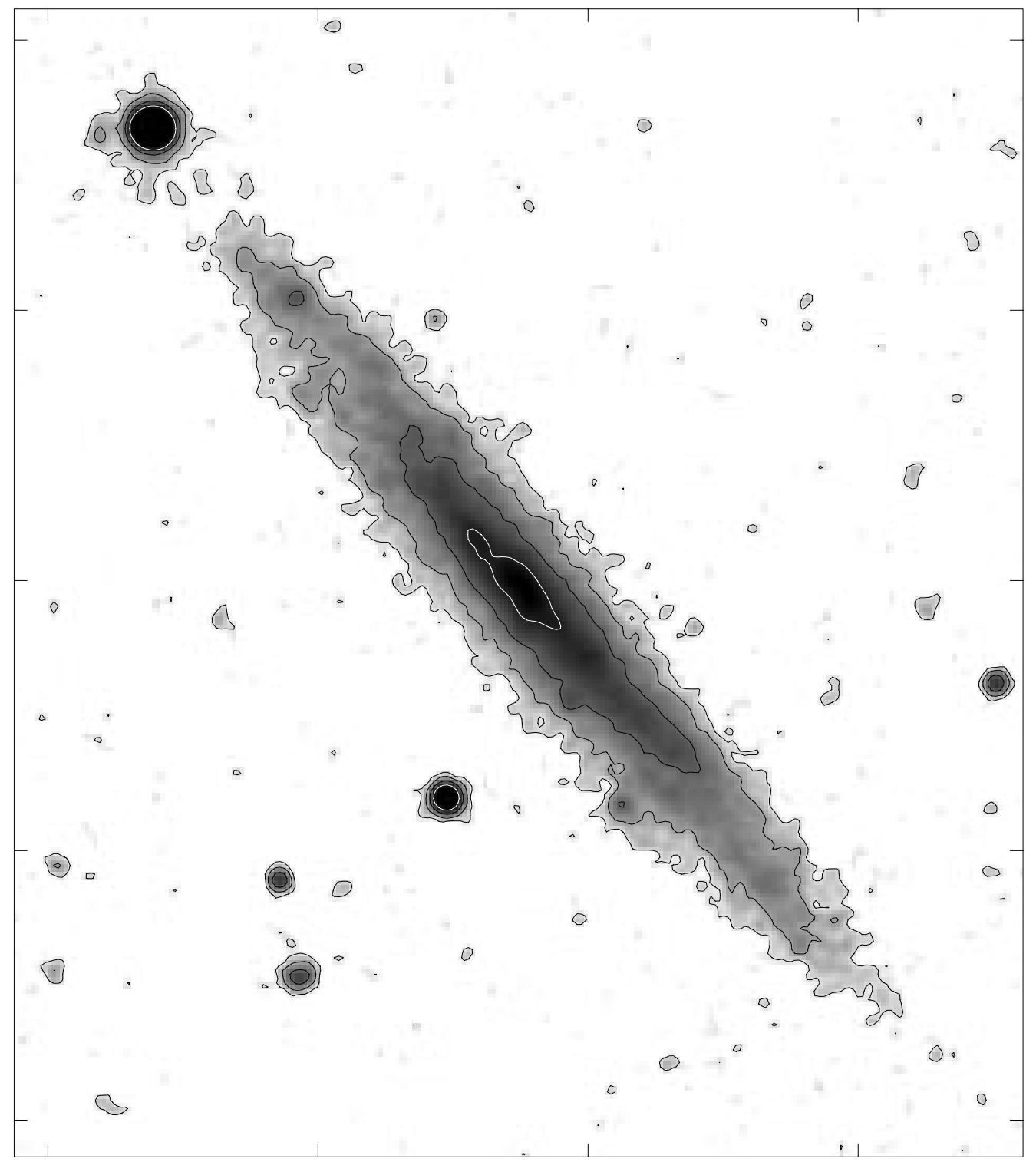

FIG. 7.-Calibrated $B$-band image of FGC 0938. Contour levels are separated by 1 mag $\operatorname{arcsec}^{-2}$. The lowest contour is $26 \mathrm{mag}$ arcsec ${ }^{-2}$. The interval between the tick marks is $20^{\prime \prime}$. North is up, and east to the left. 


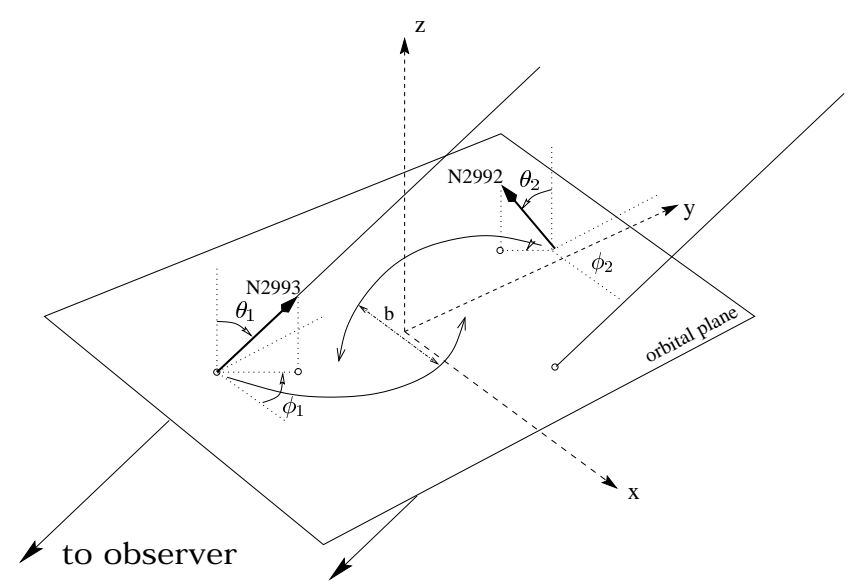

FIG. 8.-Sketch of the adopted orbital geometry for our simulations of the system Arp 245. We set up the collision in such a way that the orbital angular momentum is parallel to the $z$-axes. The initial galaxy positions are chosen such that the galaxies would reach their minimum separation on the $x$-axis, if they were point masses moving on Keplerian orbits. The orientations of the spin vectors of the two disks are specified in terms of ordinary spherical coordinates $\left(\theta_{1}, \phi_{1}\right)$, and $\left(\theta_{2}, \phi_{2}\right)$, respectively. We shall assume that NGC 2993 is seen exactly face-on and is rotating clockwise; then the line of sight is along the spin vector of NGC 2993.

Maps of the integrated $\mathrm{H}$ I distribution at a resolution of $25^{\prime \prime}$ overlaid on a $V$-band and on an $\mathrm{H} \alpha$ image of the system are shown in Figure 2 and in Figure 3, respectively. In addition to the two main galaxies, $\mathrm{H}$ I emission is clearly associated with the tidal dwarf galaxy to the north of NGC 2992, at the tip of the tidal arm. It is further traced along the northern tidal arm and in the bridge between NGC 2992 and NGC 2993. Surprisingly, there is a huge ringlike H I structure extending to the southeast of NGC 2993. In addition to emission from the NGC 2992/93 system, a strong signal is picked up from what turns out to be a companion object, known as FCG 0938 and located to the southwest. Total $\mathrm{H} \mathrm{I}$ fluxes of the galaxies are $18.6 \mathrm{Jy} \mathrm{km} \mathrm{s}^{-1}$ for the interacting system, corresponding to an $\mathrm{H}$ I mass of $4.2 \times 10^{9} M \odot$. For the companion we find $3.1 \mathrm{Jy} \mathrm{km} \mathrm{s}^{-1}$ which corresponds to an $\mathrm{H}$ I mass of $7.0 \times 10^{8} M \odot$.

The VLA integrated H I spectrum of NGC 2992 matches well, both qualitatively and quantitatively, with the singledish H I spectrum obtained by Mirabel \& Wilson (1984) at Arecibo. After beam corrections, the fluxes differ by less than $10 \%$.

\subsection{CO Observations}

$\mathrm{CO}(1-0)$ and $\mathrm{CO}(2-1)$ spectra toward the northern tidal tail of Arp 245 were obtained with the IRAM $30 \mathrm{~m}$ antenna in 1999 June. These observations are presented in Braine et al. (2000). The CO emission over the entire system, including NGC 2992, NGC 2993, and the tidal features, has subsequently been mapped in 1999 November with the SEST 15 $\mathrm{m}$ antenna at la Silla. Details regarding those observations will be given in Paper II.

\section{RESULTS}

\subsection{Status of the Interaction}

\subsubsection{The Galaxies Involved}

Table 3 summarizes the global properties of all members of the system involved in the interaction: the two spirals of moderate luminosity, NGC 2992 and NGC 2993, and the dwarf galaxy FGC 0938.

The two galaxies NGC 2992 and NGC 2993 have radial velocities that differ by less than $100 \mathrm{~km} \mathrm{~s}^{-1}$ and suffer a strong tidal interaction. NGC 2992 is a disturbed Sa spiral seen nearly edge-on at an inclination of $70^{\circ}$. On top of having an active Seyfert 1.9 nucleus and a perturbed morphology due to the interaction, NGC 2992 shows a number of peculiarities. A prominent dust lane crosses most of the galaxy from the northeast to the southwest (see Fig. 1). It could be the remnant of a past merger. The $\mathrm{H}$ I distribution is rather complex. On top of the expected rotating disk, fast moving clouds are observed. Finally, the galaxy exhibits several ionization filaments that are located well beyond the ionization cone associated with the AGN. They can be seen in Figure 3. Their kinematics is highly suggestive of outflows. Paper II will be devoted to the study of these phenomena, which are likely more directly related to the nuclear activity than to the interaction.

NGC 2993, a face-on Sab, is situated at about $3^{\prime}(27 \mathrm{kpc})$ to the southeast. On a true-color image (see Fig. 1), NGC 2993 appears much bluer than its companion. Its blue color index $(B-V=0.3 \mathrm{mag})$ and its rather high far-infrared to blue luminosity ratio $\left(L_{\mathrm{FIR}} / L_{B}=1.7\right)$ is suggestive of an active starburst. The $\mathrm{H}_{2}$ content is, however, rather low (Sofue et al. 1993) and the far-infrared to $\mathrm{H}_{2}$ mass - a measure of the star formation efficiency - is much higher than in classical starburst galaxies (Sanders \& Mirabel 1985). Active star-forming regions, as traced by $\mathrm{H} \alpha$, are present all over the galaxy (see Fig. 4), but they are more concentrated in the clumpy inner regions where the $\mathrm{H} \mathrm{I}$ peaks as well. Some red unresolved stellar clumps can be seen in the outskirts of the galaxy, especially to the north. They might be old globular clusters.

Our VLA map (Fig. 2) disclosed a third partner at roughly the same velocity, lying to the southwest, which is listed in the Catalog of Flat Galaxies (Karachentsev, Karachentseva, \& Parnovskij 1993) as FGC 0938. On our CCD image (Fig. 7), it appears as an almost undisturbed edge-on disk with an ellipticity of 0.83 , consistent with an inclination of $80^{\circ}$. With $M_{B}=-15.5$, FGC 0938 has the absolute blue magnitude of a dwarf galaxy. Its projected blue central surface brightness is $22.5 \mathrm{mag} \operatorname{arcsec}^{-2}$. Its rotating $\mathrm{H}$ I disk looks regular apart from perhaps a small extension to the west. The peak rotational velocity is of order $60 \mathrm{~km} \mathrm{~s}^{-1}$, corroborating its classification as a dwarf galaxy. Despite their different environments, FGC 0938 has many properties in common with the superthin galaxy UGC 7321 recently studied by Matthews, Gallagher, \& Van Driel (1999). It would be remarkable if a galaxy involved in a tidal interaction would manage to keep a flat disk. This is a strong indication that FGC 0938 is presently heading for its first encounter, falling toward the NGC 2992/93 system.

\subsubsection{The Tidal Features}

Arp 245 features three major tidal structures resulting from the interaction between NGC 2992 and NGC 2993: two long tidal tails escaping from both spiral galaxies and a bridge between them. Their properties are summarized in Table 4. The northern tail associated with NGC 2992 is about $16 \mathrm{kpc}$ long, a rather modest extent compared to the $100 \mathrm{kpc}$ long antennae in the prototypical interacting systems NGC 4038/39 and NGC 7252. This might be due to the smaller size and earlier morphological type of the pro- 

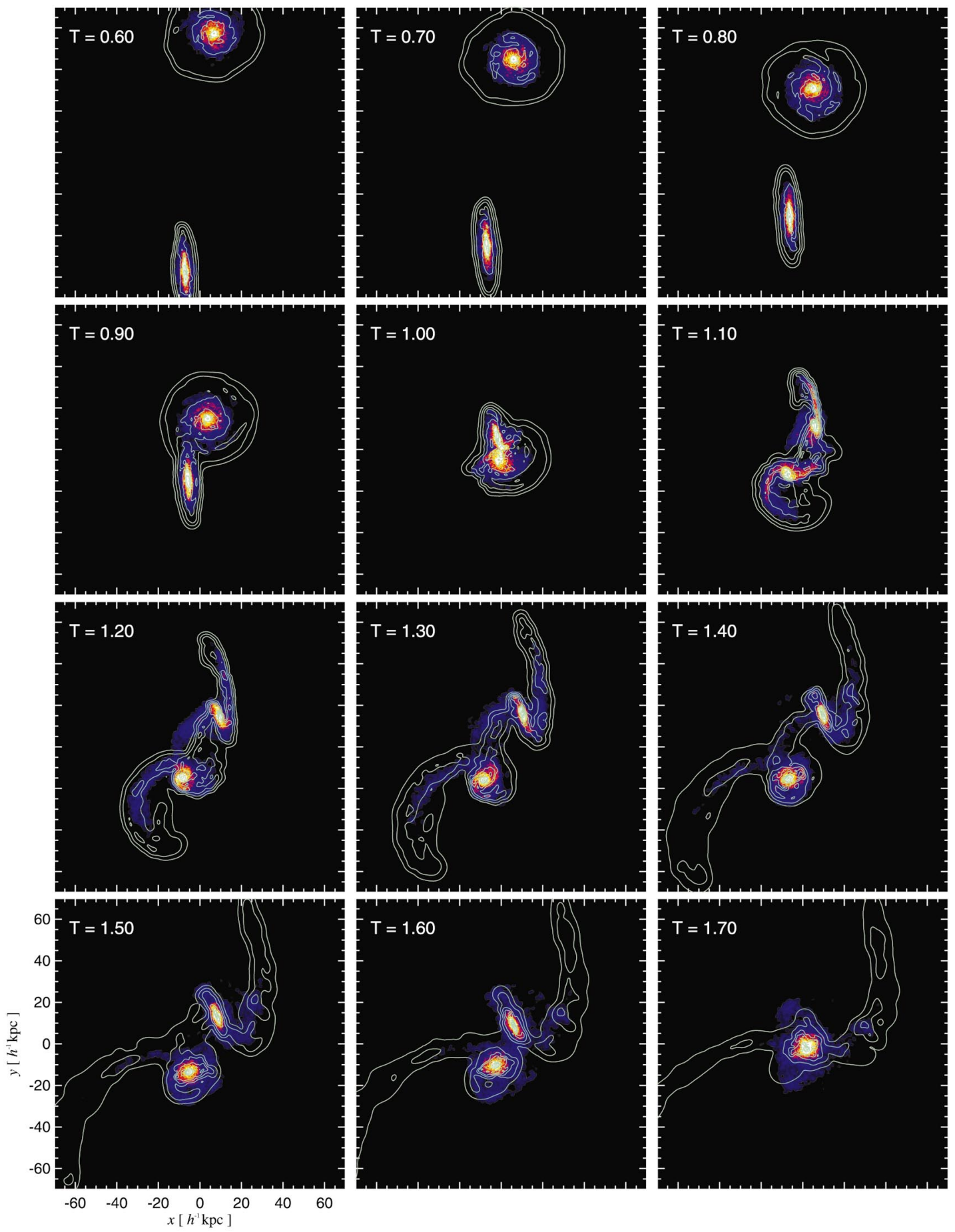

FIG. 9.-Time evolution of our numerical model of the system Arp 245. In each panel, we show the projected distribution of the stars (color scale) and that of the gas (green contours). Each panel is $140 \mathrm{~h}^{-1} \mathrm{kpc}$ on a side, and the labels give the elapsed time after the start of the simulation in units of $0.1 \mathrm{Hubble}$ times (or $\left.0.98 h^{-1} \mathrm{Gyr}\right)$. The model is shown in the plane of the sky and the orientation is the same as in Fig. 2.

genitor and to the fact that the interaction is witnessed in its early phase when the tails did not have time to fully develop. The northern tail has roughly the same distribution and extent in both its stellar (optical) and gaseous (H I) components. The optical tail looks like a plume, spreading at its tip where the $\mathrm{H}$ I peaks at a column density of $2 \times 10^{21} \mathrm{~cm}^{-2}$. This substructure, detected in all optical and near-infrared bands, suggests the formation of a tidal dwarf galaxy. It hosts several star-forming regions, clearly visible in the $\mathrm{H} \alpha$ map (see Fig. 16) at the location of the $\mathrm{H} \mathrm{I}$ clump. The integrated colors of the tail as a whole
$(B-V=0.57, V-K=0.42)$ are similar to the colors of the outer regions of NGC $2992(B-V=0.62, V-K=0.43)$. Some slightly bluer colors by $0.1 \mathrm{mag}$ are measured within the $\mathrm{H}$ II regions. The stellar population of the tail is hence dominated by old stars pulled out from the parent galaxy with some weak contribution from young stars born in situ in the tidal object. The TDG candidate will be studied in more detail in $\S 3.3$.

A diffuse stellar bridge, with a maximum $V$ surface brightness of $24 \mathrm{mag} \operatorname{arcsec}^{-2}$, connects the two spiral galaxies. The orientation of its $\mathrm{H}$ I counterpart seems to be 
slightly twisted. No stellar clusters or $\mathrm{H}$ II regions or $\mathrm{H}$ I substructures are found there. Instances of star-forming bridges do exist (e.g., NGC 6845; Rodrigues et al. 1999) but are in general quite rare among interacting systems.

The tail emanating from NGC 2993 to the east is highly curved in the plane of the sky. This is particularly visible in its $\mathrm{H}$ I component which shapes like a ring. The projected total extent of the NGC 2993 tail is $30 \mathrm{kpc}$. The optical tail appears sharp edged at its base and more diffuse farther out. The southern and western parts of the $\mathrm{H}$ I ringlike structure have no optical counterparts up to a limit of $\mu_{V}=25 \mathrm{mag}$ $\operatorname{arcsec}^{-2}$. No obvious H II regions are found along the tail, at least over the area covered by our $\mathrm{H} \alpha$ image. Further out, the absence of bright optical condensations in the broadband images strongly suggests that star formation, if any, is very weak there, contrary to the NGC 2992 tail. The main difference between the two tidal features is their respective $\mathrm{H}$ I column density which turns out to be much lower in the NGC 2993 tail. The few H I condensations scattered over the ring have all peak column densities lower than $3 \times 10^{20}$ $\mathrm{cm}^{-2}$. Surprisingly, the global color of this quiescent tidal feature $(B-V=0.39)$ is bluer than that of the northern star-forming tail and similar to that of the outskirts of its parent galaxy, NGC 2993, a starburst spiral. The blue color of the tidal tail might therefore reflect the past star-forming activity in the parent galaxy.

Our VLA map revealed one more feature with, perhaps, a tidal origin and lying east of the NGC 2993 ring, a detached $\mathrm{H}$ I cloud with a mass of $6.5 \times 10^{7} M \odot$. This object, visible in several VLA channels and therefore likely real, has no optical counterpart.

In total the $\mathrm{H}$ I in tidal features contributes to about $45 \%$ of the total H I mass measured in Arp 245. The H I mass of the northern tail is about $60 \%$ of that of NGC $2992^{16}$ whereas the $\mathrm{H}$ I mass of the southern ring is $80 \%$ of that of NGC 2993. Such large values are typical of gas-rich interacting systems where the contribution of the tails may reach 90\% (such as in Arp 105; Duc et al. 1997). On the other hand the tidal features contain a much weaker proportion of stars. The tail associated with NGC 2992 and NGC 2993 contains respectively $17 \%$ and $13 \%$ of their stellar luminous population. Such values have been determined from the $V$-band luminosities, assuming that the $M / L$ ratio is the same for all objects.

The two-dimensional kinematics of the southern tail derived from the $\mathrm{H}$ I data cube is quite smooth (see the channel map in Fig. 6 and the velocity map in Fig. 11). The ring shows a coherent velocity field, which is matched by our simulations (see below), as does the northern tail just south of the tidal dwarf. We will show in $\S 3.3 .6$ that the kinematics of the TDG itself is probably decoupled from that of its host tail.

\subsubsection{Other Unrelated Objects}

Weedman (1971) reported the presence of a blue stellar object, Weedman 2 or 0943-1403 (Bowen et al. 1994), near the tip of the tidal plume of NGC 2992. Based on lowresolution spectroscopic data, Burbidge et al. (1972) claimed that it could be a QSO somehow "associated" with NGC 2992. We obtained in 2000 January with EFOSC2 at the

\footnotetext{
16 The mass of the H I clouds seen in absorption toward the nucleus of NGC 2992 has not been included.
}

ESO $3.6 \mathrm{~m}$ telescope a spectrum of this object. It turns out to be a star. During the same run, we measured the redshifts of two uncataloged background galaxies that are situated north of NGC 2992 at, respectively, $\alpha=09^{\mathrm{h}} 45^{\mathrm{m}} 45^{\mathrm{s}} .55$, $\delta=-14^{\circ} 16^{\prime} 23^{\prime \prime} 7$ and $\alpha=09^{\mathrm{h}} 45^{\mathrm{m}} 51^{\mathrm{s}} .14, \delta=-14^{\circ} 15^{\prime} 43^{\prime \prime} .8$ (J2000.0). Both lie at a redshift of $z=0.111$.

\subsection{A Numerical Model of the Interaction}

In this paper, we are mainly interested in a first, approximate model of the NGC 2992/93 system, capturing its essential features while not necessarily providing a perfect fit to all its details. In a future paper, we plan to systematically refine this model and improve on its ability to fit the finer features of the system. The model developed here is particularly useful to date the various phenomena observed in Arp 245, to reconstruct its history and to predict its ultimate fate.

\subsubsection{Parameters of the Collision}

The first encounter models based on restricted $N$-body simulations date from almost thirty years ago, when Toomre \& Toomre (1972) were able to approximately match the morphology of four well-studied interacting systems. Since then, numerical methods have become much more sophisticated, but the number of interacting systems with tidal tails that have been modeled in a self-consistent way with numerical simulations is still small (see e.g., Salo \& Laurikainen 1993; Thomasson \& Donner 1993; Mihos, Bothun, \& Richstone 1993; Hibbard \& Mihos 1995). The main reason for that is the huge parameter space spanned by the numerous free parameters of any such model (for instance, those defining the orbital plane), precluding simple methods to find a matching solution for an observed system. Moreover the parameters describing the structure of the colliding galaxies might not be well constrained by the observations. In that respect, Arp 245 appears as a particularly attractive system to model. The morphology and dynamics of each of the interacting partners are indeed fairly well known. The large parameter space of possible collision scenarios can thus be reduced drastically by making reasonable assumptions and educated guesses about the colliding galaxies.

A sketch of the adopted geometry for our simulations is shown in Figure 8. Coordinates where the orbital plane coincides with the $x, y$ plane have been used while the orientations of the spin vectors of the two disk galaxies have been specified in terms of ordinary spherical coordinates $(\theta, \phi)$.

The nearly face-on orientation of the disk of NGC 2993, within $20^{\circ}$, and the orientation of its tidal arm emanating in the northwest suggest that its spin vector points into the plane of the sky. We have therefore identified the plane of the sky with the plane of the disk of NGC 2993, i.e., the observer is located in the opposite direction of the spin vector of NGC 2993. The disk of NGC 2992 is seen about $70^{\circ}$ inclined to the plane of the sky with the upper east side being closer to the observer (Chapman et al. 2000). Hence we have assumed that the spin vectors of the disks of NGC 2992 and NGC 2993 enclose exactly an angle of $70^{\circ}$ with each other. The prominence of both tidal tails suggests a largely prograde encounter of the two galaxies, with the vector of the orbital angular momentum presumably lying somewhere within the cone spanned by the two spin vectors, or at least relatively near to it. To further simplify things, we have here assumed that the orbital angular 

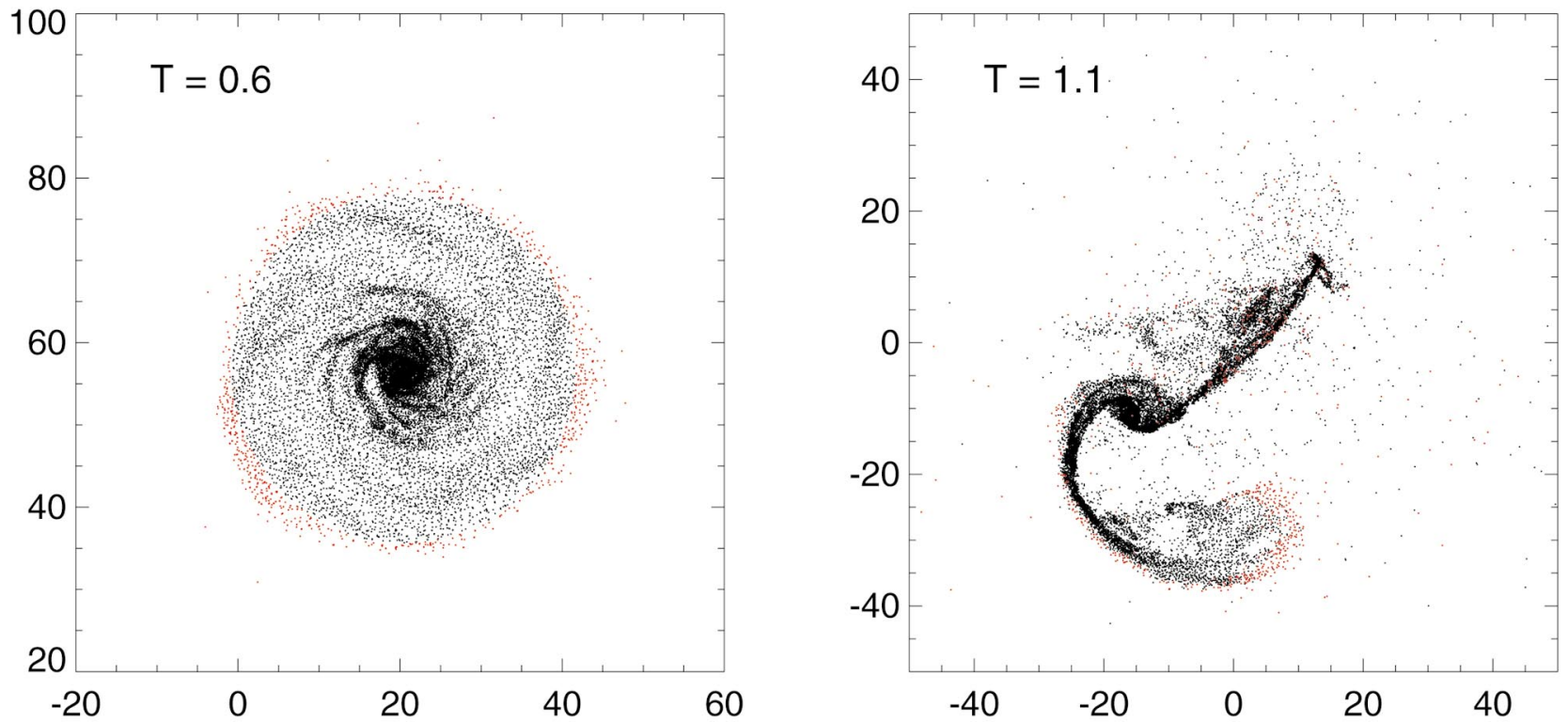

Fig. 10.-Face-on projection of the numerical model of NGC 2992 at $T=0.6$ and $T=1.1$. The $5 \%$ gas particles that are at the edge of the unperturbed disk at $T=0.6$ are shown in red. At $T=1.1$ they concentrate at the end of the tidal tail at the location of the TDG.

momentum lies in the plane spanned by the two spin vectors.

The velocity field shows that the center of NGC 2992 and its northern tail is blueshifted with respect to NGC 2993. Also note that the well-developed tails of the two galaxies indicate that the system is seen after its first encounter (as will be confirmed below). In this phase, NGC 2992 and its tail can be expected to move toward the negative $y$-axes. The tidal response of the stellar disk of NGC 2993 seems relatively weak compared with that of NGC 2992, which is in the process of forming a dwarf galaxy out of its tidal debris. This suggests that NGC 2992 is colliding almost fully prograde. Finally, we have restricted ourselves to parabolic, zero-energy encounters. The angular momentum of the orbit has been expressed in terms of the minimum separation $b$ of a corresponding Keplerian collision.

The galaxies themselves were modeled with a massive and extended dark halo with an adiabatically modified NFW profile, an embedded exponential stellar disk, a stellar bulge, and a gas distribution in the disk (see details on the numerical representations of these models in Springel \& White 1999). Note that in many observed disk galaxies the $\mathrm{H}$ I gas is substantially more extended than the exponentially distributed stars. To be able to follow the gas at large radii, we have split the available gas into two components of equal mass, one distributed just like the stars and one forming a more extended component with a constant surface mass density.

\subsubsection{Numerical Simulations}

Using fully self-consistent simulations carried out with the Tree-SPH code GADGET, we have coarsely explored the parameter space remaining under the above assumptions until a best-matching solution was found. More specifically, the numerical model presented below is specified by the following parameters. The virial velocities of the two galaxies were set to $V_{c}=120 \mathrm{~km} \mathrm{~s}^{-1}$; i.e., each of them has a total mass of $4.0 \times 10^{11} h^{-1} M_{\odot}$. We have assumed that a fraction $m_{d}=0.05$ of the total mass resides in the disk, $30 \%$ of it in the form of gas, the rest as collisionless stars. A fraction $m_{b}=0.016$ of the total mass has been put into a central stellar bulge. Adopting a spin parameter ${ }^{17}$ of $\lambda=0.06$, the resulting exponential scale length of the disks is $R_{d}=2.7 h^{-1} \mathrm{kpc}$. Half of the gas has been distributed in the disk like the stars, and the other half in a disk of radius $8 R_{d}$ with constant surface mass density. For the orbit of the collision, we adopted the parameters $\theta_{1}=50^{\circ}, \phi_{1}=90^{\circ}$, $\theta_{2}=20^{\circ}, \phi_{2}=270^{\circ}$, and $b=4 h^{-1} \mathrm{kpc}$ (see Fig. 8). The numerical simulation shown here used 60,000 particles for the dark matter, 40,000 for the stellar disk, 10,000 for the stellar bulge, and 40,000 for the gas. Cooling of gas, star formation and supernova feedback were modeled as in Springel (2000). The cooling function used to describe radiative dissipation effectively cuts off at $10^{4} \mathrm{~K}$, when the gas becomes neutral. Some of the gas can reach somewhat lower temperatures by adiabatic expansion. Star formation is assumed to convert gas into collisionless stellar material at a rate given by a simple Schmidt-type law, and supernovae feedback is modeled by an effective turbulent pressure term. A full description of the thermodynamic properties of this model is given in Springel (2000).

The simulation begins when the galaxies' dark halos just start to touch, i.e., at a separation of $240 h^{-1} \mathrm{kpc}$. It then takes about $1 h^{-1}$ Gyr to reach the perigalacticon for the first time, when strong tidal forces eject stars and gas out of the disks. This material forms the pronounced bridges and tails in Arp 245 when the galaxies separate again. Eventually, they fall back together for a second encounter, which takes place about $700 h^{-1} \mathrm{Myr}$ after the first collision. One hundred Myr later the galaxies completely coalesce and form a single merger remnant. This time evolution of the system is shown in Figure 9.

From Figure 9, it is clear that we observe the system NGC 2992/93 at a time between the first and second

\footnotetext{
${ }^{17}$ The spin parameter $\lambda$ is defined as $\lambda=L|E|^{1 / 2} / G M^{5 / 2}$, where $L$ is the angular momentum, $E$ the total energy, and $M$ the mass of the galaxy.
} 

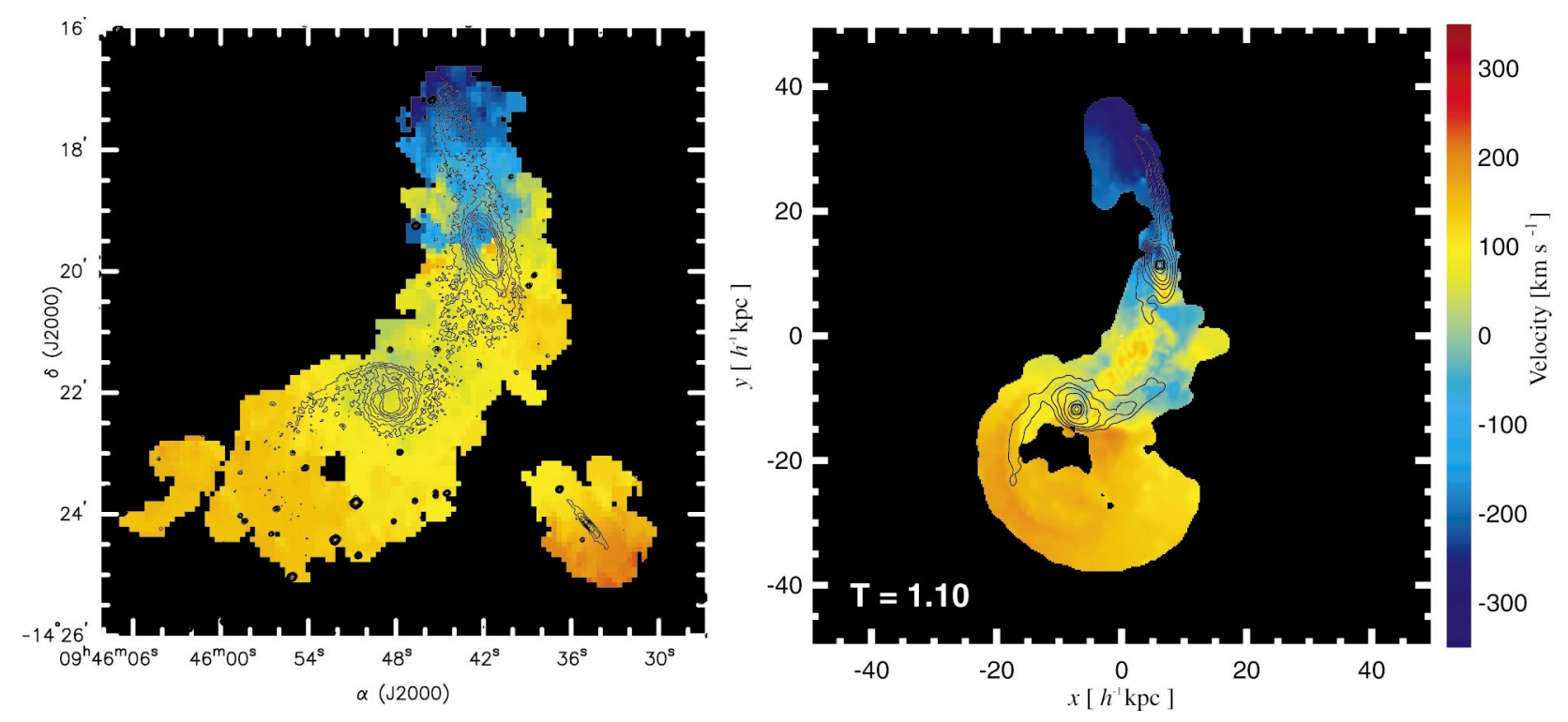

FIG. 11.-Velocity map of Arp 245. Left: H I velocity map with optical contours superposed. Right: Velocity field of our numerical simulation. The contours indicate the distribution of the stellar component. The simulation is shown at a time when it best matches the observed morphology and velocity field of Arp 245. This point of time is $100 h^{-1} \mathrm{Myr}$ after first passage through pericenter, and $1.1 h^{-1} \mathrm{Gyr}$ after the start of the simulation. The color scale gives the line-of-sight velocity of the gas relative to the systemic velocity of the system.

encounter. In this phase of the collision, the tidal features are very well defined, whereas they are going to be much more diffuse at later stages of the collision, which is a general result from simulations of other systems. Our numerical simulation provides the best match for the morphology of the system at a time of around $100 h^{-1}$ Myr after the first encounter, i.e., $1.1 h^{-1} \mathrm{Gyr}$ after the start of the simulation. ${ }^{18}$ Figure 10 shows the face-on projection of the numerical model at this time. After matching the morphology it becomes of course interesting to study the velocity field of the gas distribution at this moment of the interaction. As Figure 11 shows, the overall pattern of gas flow is well reproduced by our model. Also, the striking ring of gas around NGC 2993 has nicely formed in our model. Most of this gas is simply stemming from the long tail which is pulled from the extended $\mathrm{H}$ I disk. The inclination of the disk relative to the orbital plane helps to curl up the tail in projection to an almost closed ring. There is actually some shocked gas from the region of the bridge that helps closing the ring.

\subsubsection{Limitations of the Model}

The simulation shown in this paper corresponds to the model which so far provided the best match to the morphology of the encounter. While we are confident that the orbital geometry is reasonably well determined in this model, the internal structure of the galaxies is less well defined and may be subject to revision when we refine the model using more detailed comparisons between the simulation and the observational data. In particular, we have here assumed that the two colliding galaxies have the same mass and the same internal structure, an assumption that is unlikely to hold in reality.

Besides, the dwarf galaxy FGC 0938 has not been taken into account. Having preserved a flat disk, this galaxy is most probably plunging into the system and has not yet

\footnotetext{
${ }^{18}$ Near perigalacticon the time step in our adaptive time-step code is of order $0.05 h^{-1}$ Myr.
}

faced its first encounter with NGC 2992/93. Therefore, this intruder has not yet affected the interaction between the two spirals much. Later on, however, this galaxy might slightly perturb the merging history of the system.

Perhaps the main shortcoming of the present model is that it does not form the tidal dwarf galaxy seen in the northern tail of NGC 2992 although there is at least some gaseous overdensity at about the right place. It remains to be seen whether the tidal dwarf can be produced by a simple encounter model like the one examined here or whether additional physics or more sophisticated collision scenarios have to be invoked.

\subsection{The Tidal Dwarf Galaxy Candidate, $A 245 N$}

In this section we will study in detail the properties of the tidal object observed at the tip of the northern tail of Arp 245. We will henceforth refer to it as A245N.

\subsubsection{Morphology and Structural Parameters}

Identifying a tidal dwarf in its host tail is obviously a difficult task. Such a problem which raises the basic question of the definition of a tidal dwarf galaxy will be addressed in $\S$ 4.1.1. In a first approach, we have isolated the TDG candidate based on a morphological criterion. We have considered as belonging to A245N all stellar and gaseous material in the tail located inside the isophote $\mu_{B}=$ $24.5 \mathrm{mag} \mathrm{arcsec}-2$, north of $\delta=-14^{\circ} 18^{\prime}$ (see Fig. 2). This region contains the bulk of the atomic and ionized gas and appears to be detached in optical images (see Fig. 1). Table 5 lists the main properties of A245N. Figure 12 presents images of $A 245 \mathrm{~N}$ at different wavelengths from the optical to the near-infrared. The tidal object has been detected in all $B V R J H K^{\prime}$ bands. With an absolute blue magnitude of $M_{B}=-17.2, \mathrm{~A} 245 \mathrm{~N}$ belongs to the bright end of the dwarf galaxy population. It is actually as luminous and as extended as the LMC. Its surface brightness profile, shown in Figure 13, has been computed in the $B$ and $R$ bands following Papaderos et al. (1996a) and(1996b). It is exponential up to a radial distance of $25^{\prime \prime}(3.7 \mathrm{kpc})$ and drops beyond. The extrapolated central surface brightness in the 

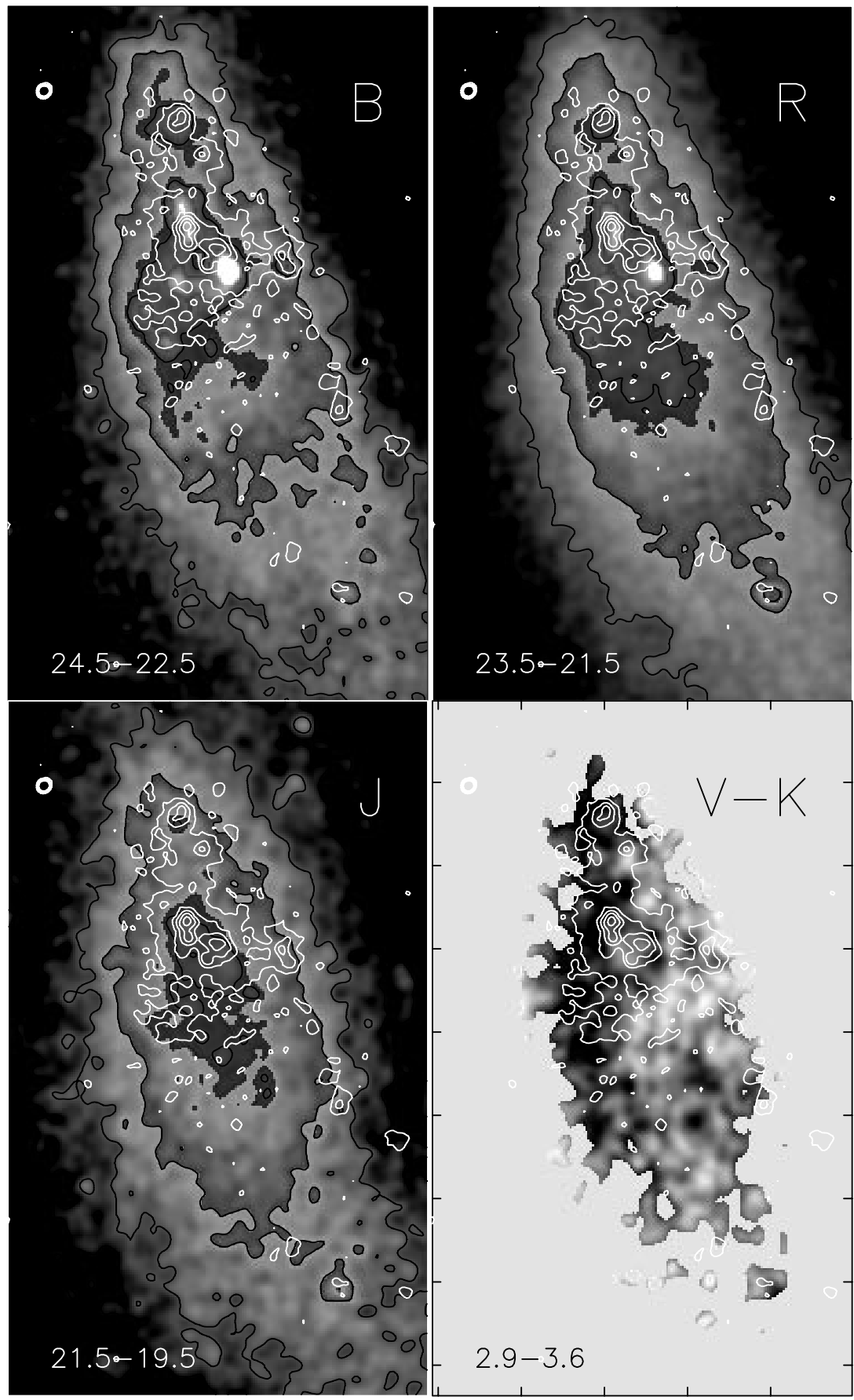

FIG. 12.- Smoothed calibrated $B R J$ images and $V-K^{\prime}$ color map of A245N. The surface brightness ranges in mag arcsec ${ }^{-2}$ are indicated at the bottom of each image. Black contours are separated by $0.5 \mathrm{mag} \mathrm{arcsec}{ }^{-2}$. The $\mathrm{H} \alpha$ contours are superposed in white. Levels are $10 \%, 30 \%, 50 \%, 70 \%$, and $90 \%$ of the peak flux $\left(4.0 \times 10^{-16} \mathrm{ergs} \mathrm{s}^{-1} \mathrm{~cm}^{-2} \operatorname{arcsec}^{-2}\right)$. Tick marks are separated by $10^{\prime \prime}$. Some stellar clumps associated with $\mathrm{H}$ II regions are clearly visible. Foreground stars have been subtracted from the images.

$B$ band is $22.4 \mathrm{mag} \operatorname{arcsec}^{-2}$ and the exponential scale length of the disk is $19^{\prime \prime}$ or $2.8 \mathrm{kpc}$. Put on the archetypal absolute magnitude versus surface brightness and scale length diagrams, A245N occupies the locus of low surface brightness dwarf irregular galaxies (see Figs. 9 and 10 in Patterson \& Thuan 1996).

\subsubsection{Stellar Populations}

Tidal tails contain two basic types of stellar populations. The first category includes stars older than the age of the interaction. They were originally formed in the parent galaxy from which they have been pulled out. Numerical simulations indicate that the stars now found at the tip of the tidal tails, i.e., in the TDGs, initially belonged to the outskirts of the parent disk. The second category is made of stars younger than the interaction formed in situ in the tails after the collapse of tidally expelled $\mathrm{H}$ I material. For nearby systems, deep color-magnitude diagrams would be the ideal tool to disentangle the first and second generation stars. At the distance of Arp 245, the galaxy can unfortunately not be resolved into stars. One has to rely on techniques based on the comparison of integrated broadband 


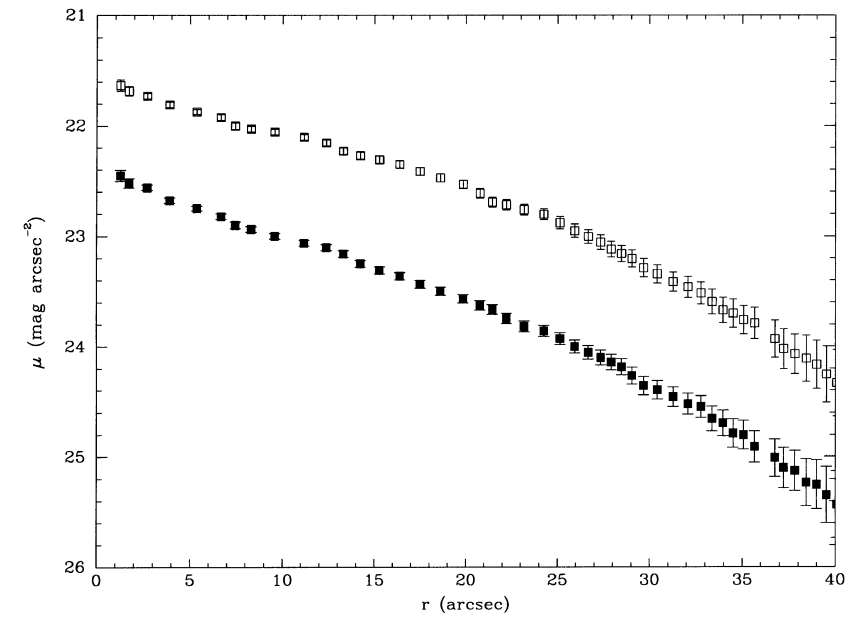

FIG. 13.- Surface brightness profile of A245N in the $B$ (filled squares) and $R$ (open squares) bands.

photometric data with predictions of evolutionary synthesis models.

As a first step toward determining the age of the populations of $A 245 \mathrm{~N}$, we have compared its optical color with that of its parent galaxy. The $B-R$ profile of both objects are displayed together in Figure 14. The external region of NGC 2992, beyond $r=30^{\prime \prime}(4.5 \mathrm{kpc})$ appears to have the same color as the tidal object except in its inner $10^{\prime \prime}$. Their spectral energy distributions remain similar over a larger wavelength range, as shown in Figure 15, which presents the SEDs of A245N and of the outskirts of NGC 2992 $\left(r>30^{\prime \prime}\right)$. Each color index differs by less than $0.1 \mathrm{mag}$, within the photometric errors. Note that the data have not been corrected for internal extinction as this is highly uncertain (see § 3.3.3). Hence, it is clear that the stellar population of A245N is currently dominated by stars pulled out from the disk of the parent galaxy.

When analyzed in detail however, the stellar population of A245N does not appear to be completely uniform. First, the object hosts several stellar clusters showing a range of colors (see Fig. 12). Their faintness and the large photometric errors prevent us from deriving from this color spread differences in ages or metallicities for the individual

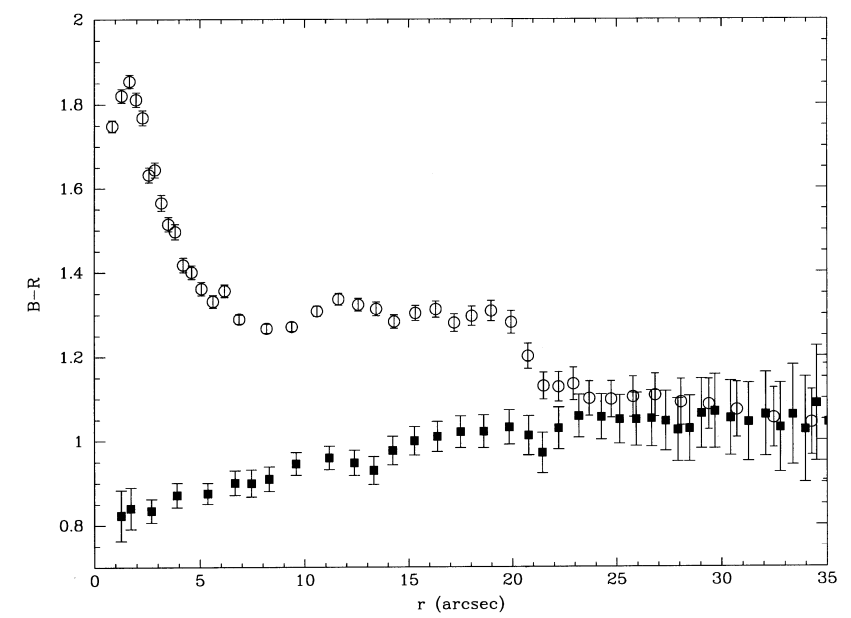

FIG. 14. $-B-R$ color profile of $\mathrm{A} 245 \mathrm{~N}$ ( filled squares) and of its parent galaxy NGC 2992 (open circles). The radius $r=0$ corresponds to the optical center of each object. clusters. ${ }^{19}$ Moreover, our spectroscopic data indicate that $\mathrm{OB}$ stars are present in A245N and therefore that the tidal object is currently forming stars. Figure 16 displays the $\mathrm{H} \alpha$ map toward A245N. Several individual clumps are concentrated in the northern region where the $\mathrm{H}$ I emission peaks. Two of the five brightest have counterparts in the broadband images (see Fig. 12) and could be associated with older star clusters. The current star formation episode weakly affects the global photometry of the galaxy. The $V-K$ color map shown in Figure 12 (where the $\mathrm{H} \alpha$ contours have been superposed) indicates that star-forming regions are bluer by about $0.1 \mathrm{mag}$ with respect to quiescent regions where no ionized gas is detected. This variation appears to be very small compared to predictions of photometric models. For a pure instantaneous starburst, the $V-K$ index might change in the first $100 \mathrm{Myr}$ by 1 to $3 \mathrm{mag}$, depending on the metallicity (Leitherer \& Heckman 1995). Taking into account the old stellar component, however, one would get a smaller color evolution.

We have estimated quantitatively the relative contribution of young to "old" stars using an evolutionary population synthesis model developed at the Göttingen observatory (Krüger et al. 1991). This code has been updated to include the bimodal star formation history of tidal dwarfs (see details in Weilbacher et al. 2000). We have only considered solar metallicities and a Scalo IMF. We have first reproduced the spectral energy distribution (SED)

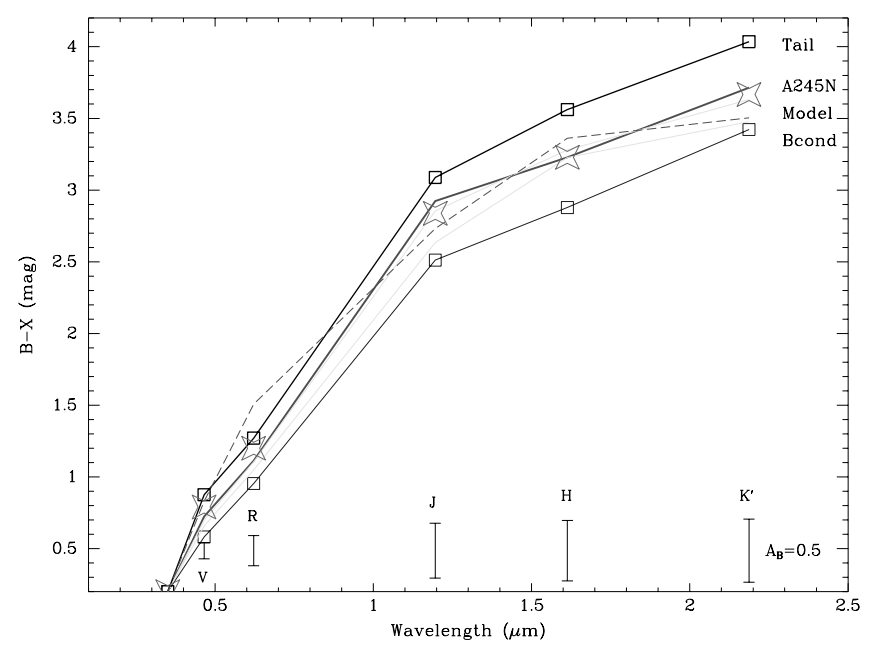

FIG. 15.- Spectral energy distribution of A245N. The $y$-axis is the $B-X$ color index in mag and the $x$-axis the wavelength in angstroms. The SED averaged over the entire TDG is traced with the thickline. The SEDs of the two brightest $\mathrm{H}$ II regions in $\mathrm{A} 245 \mathrm{~N}$ are indicated with the thin continuous lines through the center of the graph. The line at the bottom corresponds to the SED of the bright blue stellar clump in A245N. The SED of a region in the tail outside the $\mathrm{H}$ II regions is indicated with the line at the top. The stars correspond to the SED of the outskirts of NGC 2992. Finally, the dashed line is our best model for the underlying stellar population of $\mathrm{A} 245 \mathrm{~N}$. The bars at the bottom correspond to an extinction of $A_{B}=0.5 \mathrm{mag}$. All magnitudes are corrected for Galactic extinction.

${ }^{19}$ Obvious bright Galactic stars have been subtracted from the images. However, some faint foreground stars could have been mistaken for stellar clusters. The brightest blue clump in the $B$-band image for which we could obtain a deep optical spectrum does not exhibit the strong emission lines expected for an $\mathrm{H}$ II region. However the detection of faint absorption lines (in particular, $\mathrm{Ca} \mathrm{H}$ and $\mathrm{K}, \lambda \lambda 3924,3968$ ) at the right velocity confirms that it is a genuine star cluster in the TDG. 

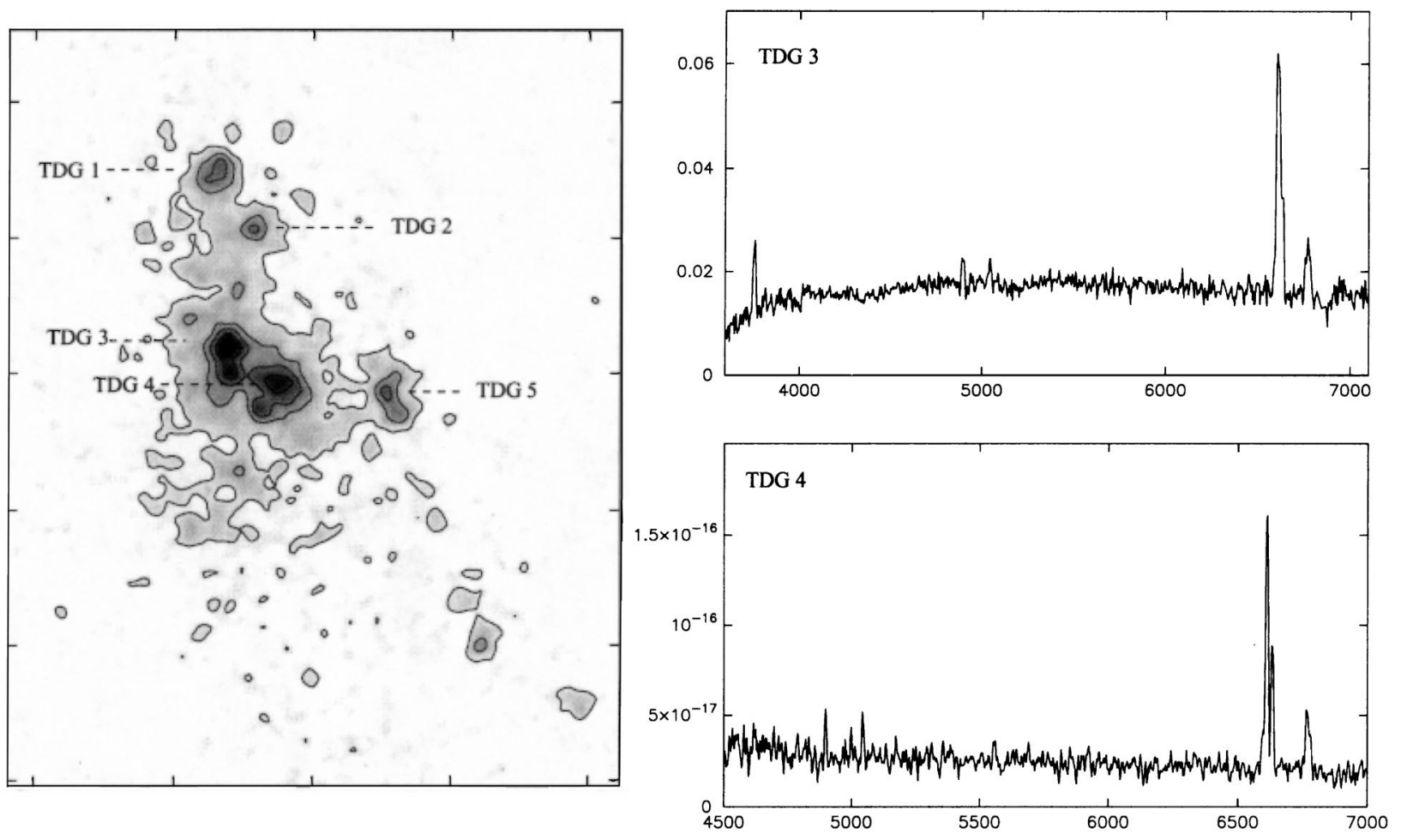

Fig. 16.-Left panel: $\mathrm{H} \alpha$ map toward A245N. The contour levels are $10 \%, 30 \%, 50 \%, 70 \%$, and $90 \%$ of the peak flux $\left(4.0 \times 10^{-16}\right.$ ergs $\mathrm{s}^{-1} \mathrm{~cm}^{-2}$ $\operatorname{arcsec}^{-2}$ ). The principal condensations are labeled. The interval between the tick marks is $10^{\prime \prime}$. Right panels: Optical spectra of the clumps TDG 3 (arbitrary units) and TDG 4 (the units are ergs cm $\mathrm{cm}^{-2} \mathrm{~s}^{-1} \AA^{-1}$ ). The spectra, typical of $\mathrm{H}$ in regions, show that the TDG is actively forming stars.

of the underlying population of $\mathrm{A} 245 \mathrm{~N}$, assuming that it is similar to the SED of the external regions of its parent galaxy, NGC 2992. The best fit, shown in Figure 15, was obtained by a template for an Sb galaxy with a characteristic age of $5 \mathrm{Gyr}$. Note that this result, which is valid for the outer disk of the spiral, is consistent with the global morphological type of the galaxy being classified as an Sa. The effect of extinction is indicated in the figure. We have then simulated an additional burst, varying its shape, strength, and duration and tried to fit the SED of the TDG candidate. Taking into account the photometric errors and the dispersion between the observed SED and the best model, we found that stars younger than $100 \mathrm{Myr}$, i.e., stars formed in situ in the tail, cannot contribute for more than $2 \%$ to the overall stellar mass of the tidal object that we estimated from our model to be $3 \times 10^{9} M \odot$. With such a low burst strength, A245N differs from most of the tidal dwarf galaxies studied so far. In particular, the southern TDG in the Arp 105 system appears much bluer than its parent galaxy; young stars could amount in this object to about $25 \%$ (Fritze-von Alvensleben \& Duc 1998) of the stellar mass. The very blue tidal dwarfs in the NGC 5291 system also seem to be completely dominated by stars formed in situ in an H I tail (Duc \& Mirabel 1998). A possible explanation for the difference between those systems and Arp 245 might be that the latter object suffered its first encounter only 100 Myr ago, which might not have been a long enough time span to go through multiple bursts of star formation in order for the young population to dominate the old one.

\subsubsection{Current Star Formation and Extinction}

We have obtained long-slit spectra of some of the $\mathrm{H} \alpha$ condensations of A245N. Our two slit positions were roughly aligned along the knots labeled TDG 1 and TDG 4 (see Fig. 16, left panel). A third spectrum (of TDG 3) was obtained during our MOS run at the ESO $3.6 \mathrm{~m}$. The spectra of TDG 3 and TDG 4 are shown in Figure 16 (right panels) and the spectrophotometric data corresponding to the knots with the highest signal to noise are listed in Table 6 . The detected emission lines have relative fluxes typical of $\mathrm{H}$ II regions ionized by young OB stars. There is no doubt that the $\mathrm{H} \alpha$ map toward the tidal dwarf traces regions of star formation. Despite the strong contribution from stars older than $100 \mathrm{Myr}$ noted before, no absorption lines are visible in the spectra. The low surface brightness of this stellar component might account for this result.

A Balmer decrement $\mathrm{H} \alpha / \mathrm{H} \beta$ of $5.7 \pm 1$ has been derived from the spectrum of TDG 4. It implies an intrinsic extinction of about $A_{B}=2.6 \pm 0.6 \mathrm{mag}$, or $E(B-V)=0.6$, a value much higher than the typical $E(B-V)=0.1$ of irregular galaxies (Hunter 1982) and about twice the value toward 30 Doradus in the LMC (Greve, Castles, \& McKeith 1991). The discrepancy could suggest a very localized obscuration in the $\mathrm{H}$ II regions and hence a clumpy dust distribution. Slightly higher absorptions have been derived in TDG 1 and TDG 3, albeit with a much larger uncertainty. Besides, $\mathrm{H}$ II regions in spiral disks also show comparable values of $E(B-V)$ (Dufour et al. 1980).

The equivalent width of the $\mathrm{H} \beta$ line in TDG 4, about $12 \pm 2 \AA$, is consistent with a starburst age of 5-7 Myr, according to the models by Cerviño \& Mas-Hesse (1994) for which an instantaneous burst with no underlying old component and a solar metallicity have been assumed. Given the age of the interaction, these newly formed stars were born in situ in the tidal tail.

We have estimated the star formation rate of the TDG from the total line emission extracted from the narrowband $\mathrm{H} \alpha$ image. The fluxes have been decontaminated for [N II] 
emission using the mean $[\mathrm{N} \mathrm{II}] / \mathrm{H} \alpha$ line ratio measured along our long-slit spectra. The calibration of Kennicutt (1998) has been used to derive the SFR from the $\mathrm{H} \alpha$ luminosity corrected for galactic extinction. It assumes a Salpeter IMF (0.1-100 M $\odot)$ and solar metallicity. We obtain an estimate of $0.03 \mathrm{M} \odot \mathrm{yr}^{-1}$. Taking into account the intrinsic extinction derived in the optical from the Balmer decrement, one would get a SFR of $0.13 M \odot \mathrm{yr}^{-1}$. The star formation rate per unit area (in $\mathrm{kpc}^{-2}$ ), $\log$ (SFR/A), is - 3.1 $(-2.5$ after correction for extinction). The normalized SFR derived by Hunter (1997) for a sample of Im galaxies covers a large range of 4 dex, between -5 and -1 , with a relative peak at -3.5 . The SFR we find for A245N is somewhat higher than this and is comparable to that of the LMC/SMC (Kennicutt \& Hodge 1986). But despite these similarities, A245N appears much redder than star-forming irregular galaxies and more typical of a spiral. The simple fact that the global photometry of the tidal dwarf does not seem to be much affected by the episode of in situ star formation implies that the latter cannot have lasted long with a constant SFR of $\sim 0.1 \mathrm{M} \odot \mathrm{yr}^{-1}$. Given the short timescale available - less than $100 \mathrm{Myr}$, a constraint set by the simulations - it is likely that the starburst started only late, perhaps less than $10 \mathrm{Myr}$ ago, as suggested by the equivalent width of the $\mathrm{H} \beta$ line.

\subsubsection{Gas Content}

A245N is a gas-rich object. Its total $\mathrm{H}$ I reservoir of $9 \times 10^{8} M \odot$ could sustain star formation episodes at rates of $0.1 \mathrm{M} \odot \mathrm{yr}^{-1}$ for several gigayears. Its $\mathrm{H}$ I mass to blue luminosity of 0.7 is typical of dwarf irregular galaxies (according to Roberts \& Haynes 1994, the median value for $\mathrm{Sm}$ and Im UGC galaxies is 0.66 ). A245N is the first tidal dwarf where molecular gas has been unambiguously found. Extended CO (1-0) emission has been detected with the IRAM $30 \mathrm{~m}$ antenna over a region of $40^{\prime \prime}$ centered on the $\mathrm{H}$ I peak (Braine et al. 2000). The $\mathrm{H}_{2}$ mass derived from the combined CO line flux is $1.4 \times 10^{8} M \odot$. Braine et al. (2000) argue that the molecular gas has formed in situ in the $\mathrm{H}$ I clouds, directly fueling the star formation regions. The very similar width of the $\mathrm{CO}$ and $\mathrm{H} \mathrm{I}$ lines supports this idea.

The star formation efficiency, i.e., the ratio of the star formation rate to the available molecular gas, $L_{\mathbf{H} \alpha} / M_{\mathrm{H}_{2}}$ is 0.006 , a value much closer to that of early-type spirals (about $0.01 L_{\mathrm{H}_{\alpha}} / M_{\mathrm{H}_{2}}$ ) than to that of irregular galaxies ( $\sim 0.05 L_{\mathrm{H}_{\alpha}} / M_{\mathrm{H}_{2}}$, Rownd \& Young 1999). Smith et al. (1999) detected molecular gas in the extended tail of the interacting galaxy Arp 215 (NGC 2782) where they derived a star formation efficiency 3 times lower than in A245N. Whereas the molecular gas in Arp 215 was found at the base of the tail and has probably been stripped from the disk of the parent galaxy, the CO detection in the tail of NGC 2992 is more directly related to $\mathrm{A} 245 \mathrm{~N}$. We have carried out a complete CO mapping of Arp 245 with the $15 \mathrm{~m}$ SEST antenna at la Silla in 1999 November. The only CO cloud detected outside the parent disks is associated with A245N at the location of the IRAM source, strongly suggesting that the $\mathrm{CO}$ found here is related to the increase in density due to self gravity in the tidal object and subsequent gravitational collapse of the tidal $\mathrm{H}$ I material.

\subsubsection{Metallicity}

We have estimated the oxygen abundance of A245N using the spectrophotometric data of the $\mathrm{H}$ II regions TDG
3 and TDG 4 listed in Table 6 . A direct determination of the abundance is not possible as the temperature sensitive $[\mathrm{O} \text { III }]_{\lambda 4363}$ line is not detected. Semi-empirical calibrations were applied instead and several diagnostic diagrams tested. We first used the approximate calibrations of Edmunds \& Pagel (1984) based on the $R 23=\left(\left[\begin{array}{ll}\mathrm{O} & \mathrm{II}\end{array}\right]_{\lambda 3727}+[\mathrm{O}\right.$ III $\left.]\right) /$ $\mathrm{H} \beta$ line ratio and applied it to TDG 3 . The relation between $R 23$ and $\mathrm{O} / \mathrm{H}$ is ambiguous as a single value of $R 23$ corresponds to two values of the oxygen abundance. However, according to McGaugh (1994), the [N II]/[O II] line ratio provides a useful diagnostic for choosing between the lower and upper branch of the $R 23-\mathrm{O} / \mathrm{H}$ relation. $\log ([\mathrm{N} \mathrm{II}] /$ [O II $>-1$ favors the highest metallicities. For TDG 3, we measured $\log ([\mathrm{N} \mathrm{II}] /[\mathrm{O} \mathrm{II}])=-0.4$, hence we selected the upper branch, and derived from $R 23$ an oxygen abundance $12+\log (\mathrm{O} / \mathrm{H})$ of $8.6 \pm 0.2$. For TDG 4 , the [O II] line is outside the instrumental wavelength range. Our estimate for this $\mathrm{H}$ II region is therefore based on the less reliable $[\mathrm{O} \mathrm{III}] / \mathrm{H} \beta$ line ratio as also calibrated by Edmunds \& Pagel (1984). We derived $12+\log (\mathrm{O} / \mathrm{H})=8.7 \pm 0.2$. However, these calibrations are not unique and depend very much on the ionization parameter $U$ (e.g., McGaugh 1991) and on the temperature of the thermal ionization source $T$. We therefore decided to run a photoionization code trying to fit all our data on TDG 3 in a consistent way. For this we used CLOUDY (Ferland 1996). Our constraints were the $[\mathrm{O} \mathrm{II}] / \mathrm{H} \beta,[\mathrm{O} \mathrm{III}] / \mathrm{H} \beta$, and $[\mathrm{N} \mathrm{III}]_{\lambda 6584} \mathrm{H} \alpha$ observed line ratios. We let the ionization parameter and temperature vary; we assumed a hydrogen density of $100 \mathrm{~cm}^{-2}$ and selected a metallicity of about one half of solar $(12+\log (\mathrm{O} /$ $\mathrm{H})=8.6$ ). With $\log (U)=-3.4$ and $T=56,200 \mathrm{~K}$, the code accurately reproduced the oxygen line intensities. The $[\mathrm{N} \mathrm{II}]_{\lambda 6584} / \mathrm{H} \alpha$ line ratio could only be obtained by increasing the relative nitrogen abundance $\log (\mathrm{N} / \mathrm{O})$ to -1.1 . This abundance is quite high compared with classical dwarf irregulars and BCDGs (Kobulnicky \& Skillman 1996) but consistent with that measured in the outer regions of spiral disks (Ferguson, Gallagher, \& Wyse 1998). Models with solar metallicity failed to reproduce the $[\mathrm{O} \mathrm{II}] / \mathrm{H} \beta$ line ratio.

The spectrophotometric study of a sample of tidal dwarf galaxies by Duc \& Mirabel (1999) indicates that TDGs have an average oxygen abundance of $Z_{\odot} / 3$ which is indepen-

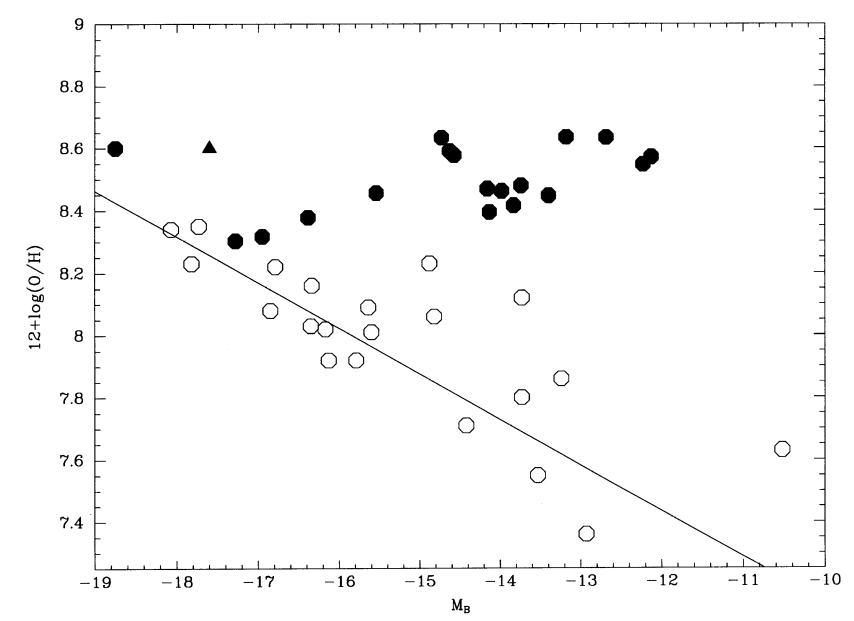

Fig. 17.-Oxygen abundance vs. absolute blue magnitude for a sample of isolated nearby dwarf galaxies (open circles, Richer \& McCall 1995) and tidal dwarf galaxies (filled circles, Duc 1995). A245N is indicated by a triangle. 
dent of their absolute blue magnitude (see Fig. 17). Made of preenriched material, TDGs are more metal rich than isolated dwarf galaxies of the same luminosity. This property may be used to identify recycled dwarf galaxies and to investigate the origin of their building material in the disk of their progenitors, as discussed in $\S 4.2$.

\subsubsection{Kinematics}

At first sight, the mean $\mathrm{H}$ I velocity field along the northern tidal tail (see Fig. 11, left panel) appears to roughly follow the model field governed by streaming tidal motions (Fig. 11, right panel). Velocities range between $2270 \mathrm{~km} \mathrm{~s}^{-1}$ at the base of the tail to $2140 \mathrm{~km} \mathrm{~s}^{-1}$ at its tip. A detailed analysis of the $\mathrm{H}$ I data cube, however, shows possible evidence of a substructure associated with $\mathrm{A} 245 \mathrm{~N}$, which is visible in position-velocity (PV) diagrams. This is best seen after rotating the $\mathrm{H}$ I data cube $\left(\alpha, \delta, V_{\mathrm{Hel}}\right)$ clockwise by $15^{\circ}$ so that the tidal tail points upward. Figure 18 presents three PV diagrams taken along a direction perpendicular to the tail axis. One cut is taken through the optical center of A245N, the other two are offset along the tail by $45^{\prime \prime}$. The cut through the tidal object shows a velocity gradient of $\sim 65 \mathrm{~km} \mathrm{~s}^{-1}$, peak to peak, extending over almost $50^{\prime \prime}$ spatially. This might suggest that part of the tidal $\mathrm{H}$ I is kinematically decoupled from its host tail showing some evidence for solid body rotation with its kinematical major axis roughly perpendicular to the tail. This component contains the bulk of the $\mathrm{H} \mathrm{I}$ in the tail (more than 60\%) and corresponds spatially to the optical tidal object. Because of the scarcity of $\mathrm{H}$ II regions in the tail, we could not determine, based on our long-slit data, any velocity profile in the ionized gas component.

Based on our low-resolution $\mathrm{H}$ I data only, we estimate a lower limit for its dynamical virial mass (i.e., uncorrected for inclination) of $9 \times 10^{8} M \odot$, identical within the errors to the $\mathrm{H}$ I mass and 3 times smaller than the estimated stellar mass. This could indicate that a large fraction of the old stellar population present in $\mathrm{A} 245 \mathrm{~N}$ does not belong to the kinematically decoupled region of the galaxy. If reliable, our low value of the dynamical mass would be consistent with a very low dark matter content, as expected for TDGs (Barnes \& Hernquist 1992).

The $\mathrm{H}$ I velocity dispersion toward the TDG candidate, $25-30 \mathrm{~km} \mathrm{~s}^{-1}$, is much higher than in a quiescent disk. The turbulence is hence dynamically important.

\subsubsection{Conclusions on the Properties of A245N}

Our detailed study of $\mathrm{A} 245 \mathrm{~N}$ indicates that the tidal object has apparently properties ranging between those of dwarf irregular galaxies (structural parameters, gas content, star formation rate) and those of spiral disks (metallicity, extinction, star formation efficiency, stellar population). A straightforward explanation would be the intrinsic hybrid nature of the galaxy which is made of disk material but results from the collapse of gaseous clouds that have masses and characteristics of dwarf galaxies.

\section{DISCUSSION}

Tidal dwarf galaxies candidates have now been found in a variety of interacting galaxies, i.e., in disk-disk systems (e.g., NGC 4038/39, Mirabel, Dottori, \& Lutz 1992), diskspheroid systems (e.g., Arp 105, Duc \& Mirabel 1994), gasrich spheroid-spheroid systems (e.g., NGC 5291, Duc \& Mirabel 1998) and advanced mergers (e.g., NGC 7252,
Hibbard et al. 1994). These interacting systems have a variety of environments: field (e.g., NGC 4038/39), compact groups (e.g., the Hickson groups, Hunsberger, Charlton, \& Zaritsky 1996) and clusters (e.g., Arp 105, NGC 5291). On the other hand, not all interacting systems with gaseous tails form dwarf galaxies, as recently shown by Hibbard \& Yun (1999). Together with the overall properties of the parent galaxies and history of the collision, the environment - i.e., density of companion galaxies, density of the intergalactic medium and strength of the associated ram pressure - might play a role in the formation and more significantly in the evolution and survival time of tidal dwarf galaxies. The exact contribution of each process is not yet known. In that respect, Arp 245 is a simple system situated in a relatively poor environment. It may hence give us some clues about the required minimum conditions for the creation of such objects. Incorporating our data on Arp $245 \mathrm{~N}$ with data from the literature on similar such systems, we will in the following review some of the outstanding questions regarding TDGs, starting with a proper definition.

\subsection{Identifying Tidal Dwarf Galaxies in Tidal Features}

\subsubsection{A Working Definition of a Tidal Dwarf Galaxy}

Luminous, star-forming knots are commonly found along the tidal features of interacting systems. Will all of them become independent from their parent galaxies and hence become true galaxies? Given their particular environments, one may doubt whether all of them will manage to survive for more than one Gyr. Therefore, in order to distinguish between such a short-lived object and a true "tidal dwarf galaxy" we need a stricter definition. We propose as a working definition for a tidal dwarf galaxy an object which is a self-gravitating entity, formed out of the debris of a gravitational interaction.

This restrictive definition ensures that TDGs are not simply agglomerated debris of collisions but that they are active objects that have their own dynamics and a potential well that is strong enough to sustain themselves against internal or external disruption for at least 1 Gyr. Such kinematically decoupled tidal objects and associated rotating gas clouds have been found in the interacting systems Arp 105 (Duc et al. 1997) and NGC 5291 (Duc et al. 1997).

\subsubsection{Possible Misidentification of TDGs}

Under the definition given above, a TDG should be considered a separate entity based on dynamical rather than morphological criteria. Tidal objects may not always look as detached or appear as contrasted entities in optical images, nor should they always have very distinct colors if they have not yet formed a substantial proportion of luminous young stars.

The true optical morphology of the galaxy might hence be hidden by an old unbound stellar component pulled out from the disk of the parent galaxy. Computing the integrated properties of a TDG, one should in principle only consider bound tidal material corresponding to the kinematically detached part of the tail. In practice, this is difficult since it would require two-dimensional high-resolution velocity data. The integrated properties of a tidal object might hence well be contaminated.

Moreover, projection effects should be taken into account. Tidal tails are generally curved (see Fig. 10) and seen edge-on; they exhibit at their apparent tip superposed material from the near and back side. The resulting project- 


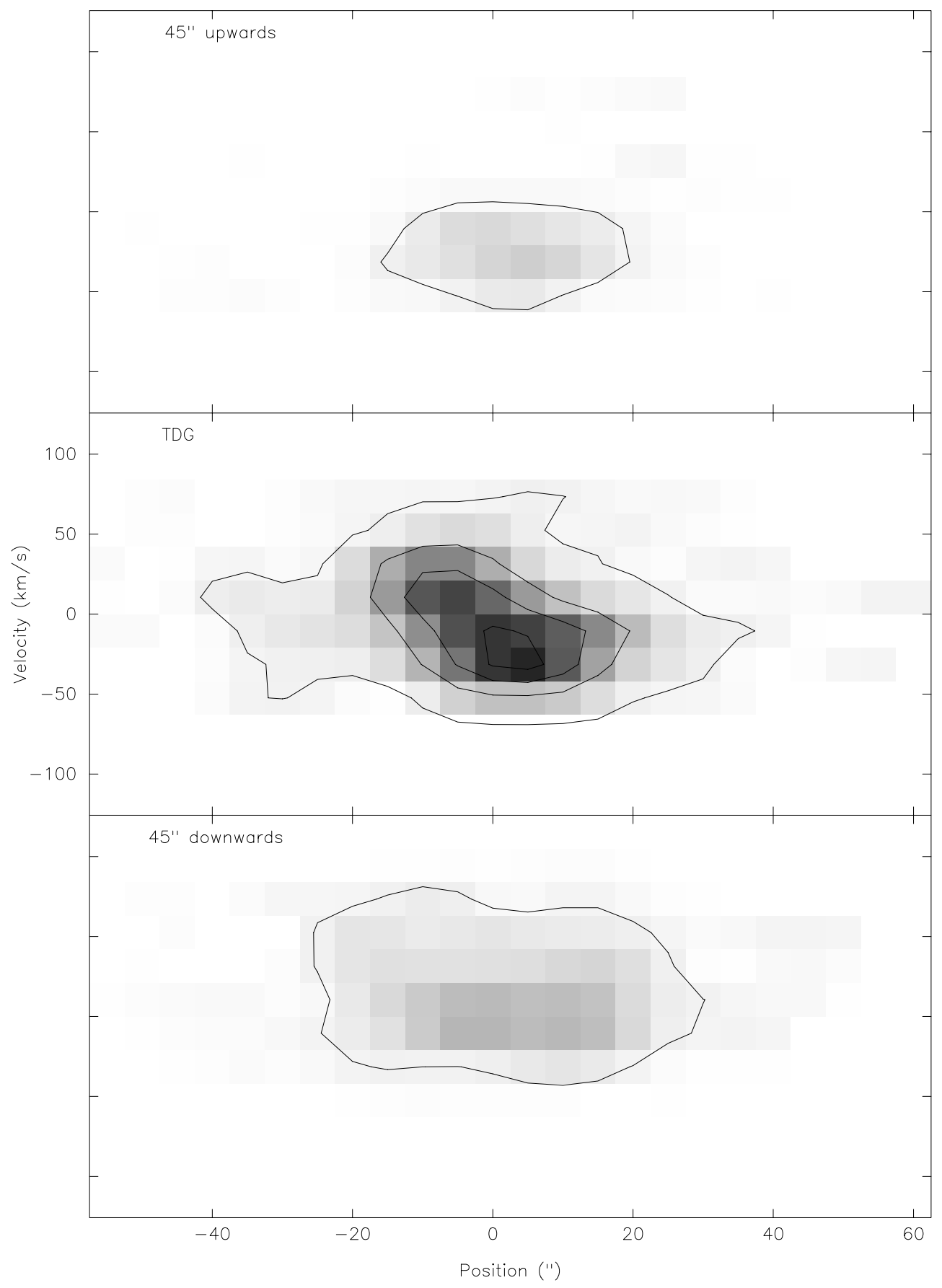

FIG. 18.-H I Position-velocity diagram at three different positions perpendicular to the northern tidal tail of NGC 2992, after rotating the galaxy clockwise by $15^{\circ}$. The central panel goes through the center of the TDG. The top and bottom ones are offset by $45^{\prime \prime}$ to the north and south, respectively. The lowest contour level is $\pm 0.5 \mathrm{mJy}_{\text {beam }}^{-1}$ and the interval is $2.3 \mathrm{mJy}^{\text {beam }}{ }^{-1}$.

ed structure might be as luminous as a dwarf galaxy and hence be mistaken as a single object. Even the streaming motions of an expending tail could mimic at its projected bend the dynamical signature of a rotating TDG (Duc et al. 1997). We could see there a range of velocities due to the contribution of several velocity vectors at different angles. It is expected, however, that, if this is the case, the resulting velocity field should be asymmetric.

Besides, some TDGs could, in principle, be mistaken with dwarf galaxies preexisting the collision, and the tail linking them to a parent galaxy would then be a bridge.

\subsubsection{Is $A 245 N$ a Tidal Dwarf Galaxy?}

Several observational facts are inconsistent with the hypothesis that $\mathrm{A} 245 \mathrm{~N}$ is a preexisting dwarf galaxy. First of all, its high metallicity is a clear sign of a recycled origin. Its colors are remarkably similar to those of the parent's outside disk. Finally, our numerical simulations show that no third body is required to reproduce the morphology of the interacting system.

The only massive tidal object in Arp 245 is found at the tip of an almost edge-on tail, a case for which projection 
effects might be important. However the $\mathrm{H} \mathrm{I}, \mathrm{H} \alpha$, and $\mathrm{CO}$ lines peak at the same location in $\mathrm{A} 245 \mathrm{~N}$ and have similar velocities. The projection hypothesis would require that all these components are rather uniformly distributed along the tails, which is not seen in the face-on eastern tail, at least for the $\mathrm{H}$ I gas. Besides, as discussed in $\S 4.3 .2$, ionized gas is only observed above a critical $\mathrm{H}$ I column density. The $\mathrm{H} \alpha / \mathrm{H}$ I coincidence is a strong indication that both phases of the gas are physically linked. It is hence more likely that the various gaseous components toward $\mathrm{A} 245 \mathrm{~N}$ form, at our spatial and velocity resolution, a single entity. The surface brightness profile of A245N, which is very well fit by an exponential profile (see Fig. 13), might suggest the presence of a stellar disk. However, the profile expected for a pure tidal tail without bound objects in it is not yet well known. In our low-resolution simulations, the SBP at the tip of the numerical tail seems to diverge from an exponential profile (see Fig. 19). Note however that the SBP measured toward A245N corresponds to that of the old stellar component pulled out from the disk of its parent galaxy. Given the young age of the interaction and the nondissipative nature of stars, it would be surprising that the stellar population is already relaxed and bound with the gaseous entity.

The kinematical independence of $\mathrm{A} 245 \mathrm{~N}$ is yet difficult to assess. $\mathrm{H}$ I position-velocity diagrams show a fairly local- ized symmetric velocity gradient reminiscent of solid-body rotation with an axis perpendicular to the tail (see $\S 3.3 .6$ ). But this is at the limits of the spatial and velocity resolution of our VLA observations. Besides, position-velocity diagrams of the simulated system computed at the same locations as the $\mathrm{H}$ I ones appear strikingly similar (see Fig. 18 and Fig. 20). Therefore, either a kinematically decoupled tidal object has already formed in our simulations, or the H I PV diagrams mostly reflect streaming tidal motions. Clearly higher resolution numerical simulations and $\mathrm{H} \mathrm{I}$ observations will shed more light on this. On the other hand, the high velocity dispersion measured in the $\mathrm{H}$ I component of $\mathrm{A} 245 \mathrm{~N}$ indicates that turbulence is almost as important dynamically as the putative rotation.

Most probably, A245N is a tidal dwarf galaxy observed in the early phases of its formation. Its gas might just be becoming self-gravitating, overcoming turbulence and streaming tidal motions. This would be consistent with the young age of the interaction.

\subsection{Origin of the TDG Building Material}

TDGs consist of material that has been pulled out from parent galaxies. But where exactly do the tidally expelled stars and gas clouds come from? Numerical simulations by Hibbard \& Mihos (1995) show that particles found at the end of the simulated tidal tails where TDGs are usually

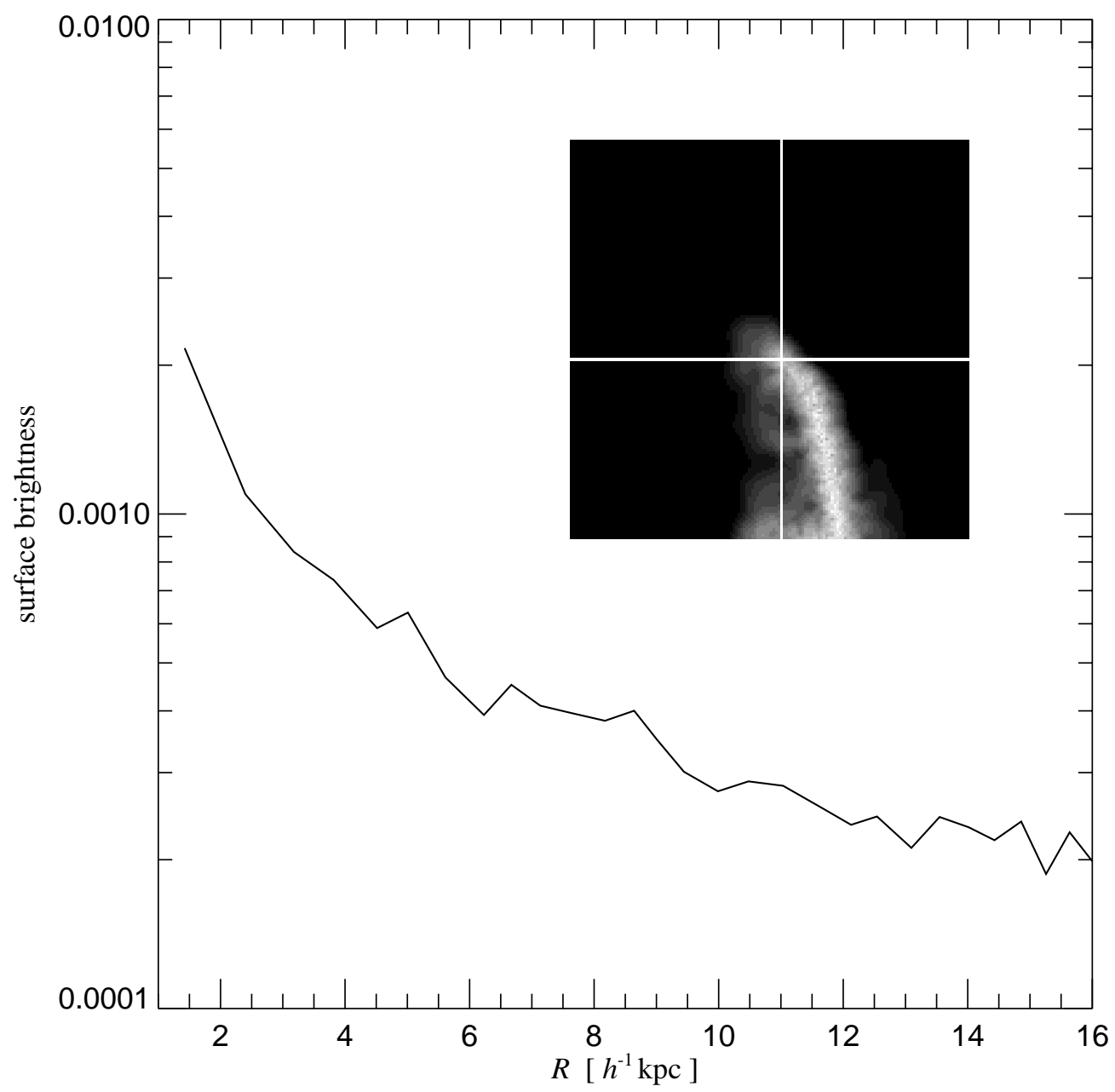

FIG. 19.- Azimuthally averaged surface brightness profile at the tip of the simulated tail at $T=1.1$. The exact center is indicated in the surface brightness map. 
observed once belonged to the outermost regions of the parent disk. This is confirmed by our numerical simulations of Arp 245 (see Fig. 10). The gas particles toward the tip of the TDG were originally in the outer gas disk, at a radius of about 8 times the exponential scale length of the stellar disk. The simulations show however that the inner parts of the tail come from regions at smaller radii in the disk.

The prediction of the simulations can be observationally checked by noting that the metallicity of TDGs reflects that of the region where their building material has come from and been preenriched. Spiral galaxies show strong metallicity gradients, from above solar in the core to one tenth of solar in the outer regions beyond the optical disk (Ferguson et al. 1998). An oxygen abundance of one third solar, the median metallicity of the ionized gas in TDGs (see Fig. 17), roughly corresponds to a radius of $R_{25}$. The slightly higher metallicity of TDG A245N suggests an even smaller galactic radius.

Hence, it is improbable that most TDGs are made up of material from much further out, unless a strong local enrichment has occurred or unless the parent galaxy had an unusual metallicity distribution prior to the collision. Enrichment of the ionized gas by a burst of star formation within a timescale of $100 \mathrm{Myr}$ or less - the maximum age of the TDG $\mathrm{A} 245 \mathrm{~N}$ as determined from numerical simulations - is unlikely. Studies of classical metal poor dIrrs and BCDGs have indeed shown that enrichment of the ionized gas does not seem to be efficient in low-mass galaxies even over longer periods. Parent galaxies with perturbed metallicity gradients, e.g., higher than normal, might be found in the class of Seyfert galaxies to which NGC 2992 belongs. Their active nucleus could pollute the interstellar medium via large-scale outflows. Whereas the absence of a steep abundance gradient has been reported by Evans \& Dopita (1987) in the prototype Seyfert 2 galaxy NGC 1068, this property was not found in other Seyfert galaxies (Schmitt, Storchi-Bergmann, \& Baldwin 1994; StorchiBergmann et al. 1996). Statistical data are so far missing to link any anomalous metallicity gradient distributions with the nuclear activity and related ejection phenomena.

Clearly, precise measurements of the metallicity distribution in the parent galaxies ${ }^{20}$ and more detailed numerical simulations of interacting systems would be required to further investigate the origin of the building material of TDGs.

\subsection{Required Conditions for the Formation of a TDG}

\subsubsection{Location of TDGs in the Tail}

Numerical simulations by Barnes \& Hernquist (1992) and Elmegreen, Kaufman, \& Thomasson (1993) suggest that TDGs should form from gravitational instabilities that grow in the debris of the collision. These objects appear as bound condensations distributed all along the tidal features. Some tails of interacting systems indeed host numerous faint blue knots showing signs of star formation (Schombert et al. 1990; Weilbacher et al. 2000). One of the two most spectacular objects of that kind are NGC 4676, the Mice (Hibbard \& van Gorkom 1996; Sotnikova \& Reshetnikov 1998), and IRAS 19254-7245, the Superantennae (Mirabel,

\footnotetext{
${ }^{20}$ This study is extremely difficult to do in NGC 2992 because of the absence of $\mathrm{H}$ II regions in its disk. Metallicity measurements are very uncertain in the ELRs of active galaxies.
}

Lutz, \& Maza 1991; Mihos \& Bothun 1998). However, systems containing substructures that comply with our definition of a TDG generally show the star formation being concentrated in a single object located at the tip of the stellar tail, such as TDG A105N (Duc et al. 1997). And this is also, of course, the case for A245N, which is located at the end of the tidal plume stemming from NGC 2992.

So, why is there only one single massive star-forming clump as soon as a TDG has been formed, and why is it located at the tip of the tidal tail?

Excluding the projection effects that could lead to a misidentification of a TDG at the tip of the tail, part of the explanation might have to do with the timescale for tidal material to fall back to the merger. This rate scales as $t^{-5 / 3}$ (Hibbard \& Mihos 1995) and is highest shortly after perigalacticon. Therefore, bound objects that might have formed at the base of the tail would have already fallen back toward their progenitor, leaving as the only viable region for the formation of a TDG the tips of tails. Modeling the prototype merger NGC 7252 with $N$-body simulations, Hibbard \& Mihos (1995) have found that about half of the tidal material situated at the base of the already formed tail (130 Myr after periapse) falls back within 130 Myr. Arp 245 is observed about 100 Myr after perigalacticon and only about $50 \mathrm{Myr}$ after the tails have developed (see Fig. 9). Over a timescale of $50 \mathrm{Myr}$, about one-fourth of the initial tidal material might have fallen back already. A more precise study of the temporal evolution of the return of both gaseous and stellar material, based on our own numerical simulations of Arp 245, will be studied elsewhere.

Another hypothesis could be that the initial clumps, proto-TDGs, might have merged. Detailed high-resolution simulations could test whether the formation of TDGs proceeds in a hierarchical way. If true, short-lived tidal features would not offer a suitable environment for the formation and survival of TDGs. In particular, tails that have a life expectancy greater than $1 \mathrm{Gyr}$ are more likely to generate TDGs than bridges which only last for about $\sim 10^{8} \mathrm{yr}$ (Struck 1999). Another obstacle faced by tidal bridges, illustrated for instance by Arp 245, is their intrinsic low surface density, a critical parameter as we will show in the next section.

\subsubsection{Conditions for Star Formation}

The fact that all observed tidal dwarfs, i.e., kinematically independent objects in tidal features, are star-forming objects is partly a selection effect: TDG candidates have generally been selected for their brightness and blue color. On the other hand, no bound tidal gas cloud is yet known which is not associated with a stellar counterpart. So the question raised in the previous section-where do TDGs form? - could be addressed by answering another one: what conditions are necessary for star formation (SF) to commence in tidal debris?

$\mathrm{H}$ I maps of interacting systems give some clues: all TDGs are found in $\mathrm{H}$ I tails and are roughly adjacent to local peaks in the H I column density map. This fact is not specific to TDGs, of course, and actually applies to SF regions in all types of galaxies, from spirals to dwarf irregulars, and might simply reflect the fact that $\mathrm{H}_{\mathrm{I}}$ is the raw material for any SF event. However, not every H I clump hosts a SF region. The onset of star formation apparently depends on an $\mathrm{H}$ I column density threshold, as first noted by Davies, Elliot, \& Meaburn (1976) for the LMC. Further 


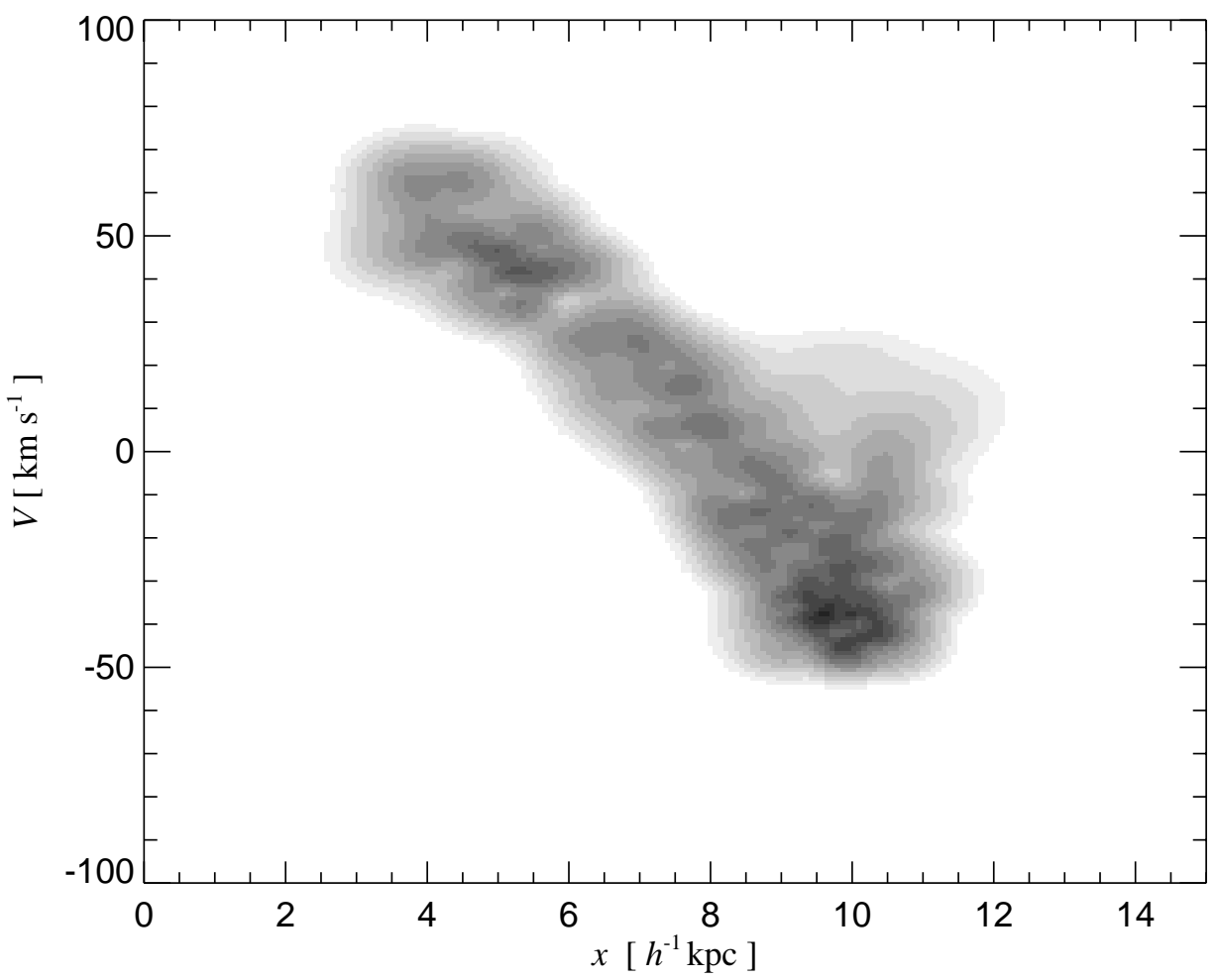

FIG. 20. - Simulated position-velocity diagram at a position perpendicular to the northern tidal tail, after rotating the $T=1.1$ projected model clockwise by $15^{\circ}$. The PV diagram has been computed in a thin slice of the gaseous component at $y=32 h^{-1} \mathrm{kpc}$.

studies of dwarf irregulars (Gallagher \& Hunter 1984; Skillman 1987; Taylor et al. 1994) and low surface brightness galaxies (van der Hulst et al. 1993; van Zee et al. 1997) suggest that this threshold is about $0.5-1 \times 10^{21} \mathrm{~cm}^{-2}$.

Neither the universality of this threshold, nor the underlying physics is known. It would be surprising if it would be the same in spiral disks, in dwarf galaxies, and in the more chaotic environment of interacting galaxies. And a word of caution, when making comparisons, one should aim for comparable linear resolution as the peak H I column density, because of beam dilution, decreases with decreasing resolution.

In the case of Arp 245 both tidal tails show a clumpy H I structure. Star formation occurs only in the TDG above densities of $2 \times 10^{21} \mathrm{~cm}^{-2}$. All condensations in the southern tail have their peak below $5 \times 10^{20} \mathrm{~cm}^{-2}$ (see Fig. 3 ) and no SF is associated with this tail. In turn, toward the spiral NGC 2993 ionized gas is observed above our sensitivity limit of $0.4 \times 10^{-16} \mathrm{ergs} \mathrm{s}^{-1} \mathrm{~cm}^{-2} \operatorname{arcsec}^{-2}$ at those locations where the $\mathrm{H}$ I density exceeds $10^{21} \mathrm{~cm}^{-2}$. The threshold hence appears to be 2 times higher in the tidal dwarf than in the spiral disk. The connection between $\mathrm{H} \alpha$ and $\mathrm{H} \mathrm{I}$ in the other spiral, NGC 2992, is more difficult to assess. Some ionization filaments are observed at large galactic radii where the $\mathrm{H}$ I has column densities well below $5 \times 10^{20} \mathrm{~cm}^{-2}$. However this apparent decoupling between the two phases of the gas is mainly due to the very nature of the ionizing source, probably hard UV radiation from the central AGN or from a shock induced process (Allen et al. 1999, Paper II).

As mentioned, the physical origin for the empirical $\mathrm{H} \mathrm{I}$ threshold is not well understood (see review by Skillman 1996). One possible explanation is a gravitational instability, as elaborated by Kennicutt (1989) in the case of spiral disks. Because of the limited resolution and the obviously strong tidal forces, it is not possible to test the validity of this explanation for TDGs.

Alternatively, the threshold in $N_{\mathrm{H}}$ might correspond to a minimum required column density of dust to shield the gas from the UV radiation field (Federman, Glassgold, \& Kwan 1979; Skillman 1987). Given sufficient shielding, a molecular cloud can form from the $\mathrm{H}$ I allowing star formation to proceed. This critical density should be metallicity dependent since the dust-to-gas ratio varies with the element abundance (Franco \& Cox 1986). It is then expected that recycled tidal dwarfs should have an $N_{\mathrm{H}}$ threshold lower than that of isolated metal-poor dwarf galaxies and similar to that of spiral disks. Table 7 lists the $\mathrm{H}$ I column density data available in the literature for several star-forming TDGs. Column (1) shows the peak $N_{\mathrm{H}}$ - or a range of values in case several TDGs are present - and column (2) the linear resolution. The majority of the TDGs so far studied have a column density of $2-3 \times 10^{20} \mathrm{~cm}^{-2}$, indeed 3 times lower than in dIrrs (Skillman 1987; Taylor et al. 1994). However, the metal rich, CO rich, and presumably dusty TDG belonging to Arp 245 has its $\mathrm{H}$ II clustered in the central core of the $\mathrm{H}$ I cloud where the $N_{\mathrm{HI}}$ exceeds $2 \times 10^{21} \mathrm{~cm}^{-2}$. Rather than stating that Arp $245 \mathrm{~N}$ is the exception, one should perhaps rather question the low star formation thresholds in the other TDGs, noting that beam dilution for the other TDGs, which were observed at much lower resolution, could partly account for this large difference.

\subsection{Models and Numerical Simulations of TDGs}

Several theoretical models for the formation of TDGs have been proposed. Elmegreen et al. (1993) suggest that massive $\mathrm{H}$ I clouds form first in the outer disk of interacting 
TABLE 7

H i Peak Column Densities in TDGs

\begin{tabular}{cccl}
\hline \hline Object & $\begin{array}{c}N_{\mathrm{H}} \\
\left(10^{20} \mathrm{~cm}^{-2}\right)\end{array}$ & $\begin{array}{c}\text { Resolution } \\
\left(\mathrm{kpc} \mathrm{beam}^{-1}\right)\end{array}$ & \multicolumn{1}{c}{ Source } \\
\hline NGC $2992 / 93 \ldots \ldots$ & 23 & $2.9 \times 2.1$ & This paper \\
NGC $5291 \ldots \ldots \ldots$ & $2.2-12.6$ & $7.3 \times 4.2$ & Malphrus et al. (1997) \\
NGC $7252 \ldots \ldots \ldots$. & $3.2-5.5$ & $8.1 \times 4.8$ & Hibbard et al. (1994) \\
NGC $4038 / 39 \ldots \ldots$ & 2.3 & $4.9 \times 16.0$ & van der Hulst (1979) \\
Arp 105 $\ldots \ldots \ldots \ldots$. & $3.2-9.6$ & $13 \times 11.7$ & Duc et al. (1997) \\
\hline
\end{tabular}

galaxies. According to the Jeans criterion, the local high velocity dispersion induced by the collision allows the formation of clouds with masses much higher than in a quieter environment, up to $10^{8} M \odot$. These clouds are then tidally expelled into the IGM where they collapse.

Wallin (1990) put forward a geometrical torsion of the tail leading to a local density enhancement. Barnes \& Hernquist (1992) propose a local amplification of preexisting stochastic clumps in the tidal tails that start accreting low $\sigma$ material - in particular gas - and become gravitationally bound. The collected material will then collapse until rotation takes over.

Numerical simulations that might help selecting between these different theories of the formation of TDGs should also take into account the observed properties of these objects and explain:

1. knots, perhaps star forming, but not necessarily leading to TDGs, along the tidal tails;

2. star formation whenever the $\mathrm{H}$ I clumps reach some critical column density, the origin of which could either be a gravitational instability within the tidally ejected gas, or shielding from the UV radiation field once clumps get dense enough;

3. that in most systems eventually only one massive object per tail forms, located at the end of it;

4. the possible merging between individual preexisting clumps;

5. the timescale for the formation of a TDG-this can be quite short: in Arp 245, a TDG developed in less than $100 \mathrm{Myr}$;

6. the $\sim \frac{1}{3}$ of solar metallicity in the TDGs;

7. the formation of TDGs in pure $\mathrm{H}$ I tails which is the case when the interaction involves gas-rich early-type galaxies from which stars are much less efficiently pulled out than the gas (e.g., in NGC 5291, Duc \& Mirabel 1998).

\section{CONCLUSIONS}

We have presented a multiwavelength study of the interacting system Arp 245 (NGC 2992/93) and a preliminary numerical simulation of the collision. From our observations and models, we obtain the following results:

1. The system is observed at an early stage of the interaction, $100 \mathrm{Myr}$ after first perigalacticon and $700 \mathrm{Myr}$ before the final merger. At the current time two long tidal tails and a bridge have developed. $N$-body/SPH simulations reproduce fairly well both the optical and $\mathrm{H}$ I features, in particular the ringlike structure observed in the gaseous component. The VLA map reveals a third member to the interacting system: an $\mathrm{H}$ I rich dwarf galaxy, seen almost edge-on and looking unperturbed. This object is hence probably falling into the group.

2. $\mathrm{H}$ I in emission is found all along the two optical tidal tails where it accounts for respectively $60 \%$ and $80 \%$ of the H I of their progenitors, NGC 2992 and NGC 2993. The H I shows a peak at the end of the NGC 2992 tail where a gas reservoir of $10^{9} \mathrm{M} \odot$ supplies a star-forming, tidal object, A245N. H I is seen in absorption toward the active nucleus of NGC 2992. Star formation, as traced by $\mathrm{H} \alpha$, occurs in the main body of NGC 2993. In the tidal features, it is restricted to the tip of the northern tail at the location of the TDG $\mathrm{A} 245 \mathrm{~N}$, where the $\mathrm{H}$ I column density is at least $1.5-2$ $\times 10^{21} \mathrm{~cm}^{-2}$.

3. The global properties of $A 245 \mathrm{~N}$ range between those of dwarf irregular galaxies as far as its structural parameters, gas content and star formation rate are concerned, and those of spiral disks as for its metallicity, extinction, star formation efficiency and stellar population. In particular, although the object is actively forming stars, and has a large atomic and molecular gas reservoir, the bulk of its stellar population is still dominated by the old component pulled out from the parent galaxy. Whereas A245N appears to be a self-gravitating entity, our data lack spatial resolution to probe its proper dynamics. Most probably, A245N is a tidal dwarf galaxy that is still in the process of formation.

We are grateful to the support astronomers and night assistants from the $2 \mathrm{p} 2$, NTT, and 3p6 teams which have helped us during our different observing runs at la Silla. Special thanks to Pierre Leisy for his precious support, to Vassilis Charmandaris who did the $\mathrm{CO}$ observations at SEST and to Polichronis Papaderos who has computed the surface brightness profiles of our objects. This work has greatly benefited from discussions with Jonathan Braine, Uta Fritze-von Alvensleben, Simon White and from the very useful comments of the referee, Curtis J. Struck. P.-A. D. acknowledges support from the network Formation and Evolution of Galaxies set up by he European Commission under contract ERB FMRX-CT96086 of its TMR program. I. F. M. acknowledges support from CONICET/Argentina. E. B. acknowledges partial support through a grant from CONACyT (No. 27606-E) This research has made use of the Lyon-Meudon Extragalactic Database (LEDA) supplied by the LEDA team at the CRAL-Observatoire de Lyon (France), as well as of the NASA/IPAC Extragalactic Database (NED) which is operated by the Jet Propulsion Laboratory, California Institute of Technology, under contract with the National Aeronautics and Space Administration. 


\section{REFERENCES}

Allen, M. G., Dopita, M. A., Tsvetanov, Z. I., \& Sutherland, R. S. 1999, ApJ, 511, 686

Alonso-Herrero, A., Simpson, C., Ward, M. J., \& Wilson, A. S. 1998, ApJ, 495, 196

Barnes, J. E. \& Hernquist, L. 1992, Nature, 360, 715

Bowen, D. V., Osmer, S. J., Blades, J. C., Tytler, D., Cottrell, L., Fan, X. M., \& Lanzetta, K. M. 1994, AJ, 107, 461

Braine, J., Lisenfeld, U., Duc, P.-A., \& Leon, S. 2000, Nature, 403, 867

Burbidge, E. M., Strittmatter, P. A., Smith, H. E., \& Spinrad, H. 1972, ApJ, 178, L43

Casali, M., \& Hawarden, T. 1992, UKIRT JCMT Newsl., No. 3, 33

Cerviño, M., \& Mas-Hesse, J. M. 1994, A\&A, 284, 749

Chapman, S., Morris, S., Alonso-Herrero, A., \& Falcke, H. 2000, MNRAS, 314, 263

Colbert, E. J. M., Baum, S. A., Gallimore, J. F., O’Dea, C. P., Lehnert, M. D., Tsvetanov, Z. I., Mulchaey, J. S., \& Caganoff, S. 1996, ApJS, 105, 75

Condon, J. J., Condon, M. A., Gisler, G., \& Puschell, J. J. 1982, ApJ, 252, 102

Davies, R. D., Elliot, K. H., \& Meaburn, J. 1976, MmRAS, 81, 89

Dubinski, J., Mihos, J. C., \& Hernquist, L. 1996, ApJ, 462, 576

Duc, P.-A. 1995, Ph.D. thesis, Univ. Paris

Duc, P.A., Brinks, E. Wink, J. E. \& Mirabel, I. F. 1997, A\&A, 326, 537

Duc, P.-A. \& Mirabel, I. F. 1994, A\&A, 289, 83 1998, A\&A, 333, 813

1999, in IAU Symp. 186, Galaxy Interactions at Low and High Redshift, ed. J. E. Barnes \& D. B. Sanders (Dordrecht: Kluwer), 61

Dufour, R. J., Talbort, R. J., J., Jensen, E. B., \& Shields, G. A. 1980, ApJ, 236, 119

Durret, F. \& Bergeron, J. 1987, A\&A, 173, 219 1988, A\&AS, 75, 273

Edmunds, M. G., \& Pagel, B. E. J. 1984, MNRAS, 211, 50

Elmegreen, B. G., Kaufman, M., \& Thomasson, M. 1993, ApJ, 412, 90

Evans, I. N. \& Dopita, M. A. 1987, ApJ, 319, 662

Federman, S. R., Glassgold, A. E., \& Kwan, J. 1979, ApJ, 227, 466

Ferguson, A. M. N., Gallagher, J. S., \& Wyse, R. F. G. 1998, AJ, 116, 673

Ferland, G. 1996, Hazy, a Brief Introduction to CLOUDY (Internal Rep.)

(Lexington: Univ. Kentucky Dept. Physics and Astron.)

Franco, J. \& Cox, D. P. 1986, PASP, 98, 1076

Fritze-von Alvensleben, U., \& Duc, P. A. 1998, in The Magellanic Clouds and Other Dwarf Galaxies, ed. T. Richtler \& J. M. Braun (Aachen: Shaker), 141

Gallagher, J., \& Hunter, D. 1984, ARA\&A, 22, 37

Gilli, R., Maiolino, R., Marconi, A., Risaliti, G., Dadina, M., Weaver, K., \& Colbert, E. J. M. 2000, A\&A, 355, 485

Glass, I. S. 1997, MNRAS, 292, L50

Gregg, M. D., \& West, M. J. 1998, Nature, 396, 549

Greve, A., Castles, J., \& McKeith, C. D. 1991, A\&A, 251, 575

Hamuy, M., Walker, A. R., Suntzeff, N. B., Gigoux, P., Heathcote, S. R., \& Phillips, M. M. 1992, PASP, 104, 533

Hibbard, J. E., Guhathakurta, P., van Gorkom, J. H., \& Schweizer, F. 1994, AJ, 107, 67

Hibbard, J. E., \& Mihos, J. C. 1995, AJ, 110, 140

Hibbard, J. E., Vacca, W. D., \& Yun, M. S. 2000, AJ, 119, 1130

Hibbard, J. E., \& van Gorkom, J. H. 1996, AJ, 111, 655

Hibbard, J. E., \& Yun, M. S. 1999, AJ, 118, 162

Hunsberger, S. D., Charlton, J. C., \& Zaritsky, D. 1996, ApJ, 462, 50

Hunter, D. A. 1982, ApJ, 260, 81

Hunter, D. A. 1997, PASP, 109, 937

Karachentsev, I. D., Karachentseva, V. E., \& Parnovskij, S. L. 1993 , Astron. Nachr., 314, 97

Kaufman, M., Brinks, E., Elmegreen, D. M., Thomasson, M., Elmegreen, B. G., Struck, C., \& Klaric, M. 1997, AJ, 114, 2323
Kennicutt, R. C., Jr. 1989, ApJ, 344, 685

1998, ARA\&A, 36, 189

Kennicutt, R. C., Jr., \& Hodge, P. W. 1986, ApJ, 306, 130

Kobulnicky, H. A., \& Skillman, E. D. 1996, ApJ, 471, 211

Krüger, H., Fritze-von Alvensleben, U., Loose, H., \& Fricke, K. J. 1991, A\&A, 242, 343

Landolt, A. U. 1992, AJ, 104, 340

Leitherer, C., \& Heckman, T. M. 1995, ApJS, 96, 9

Malphrus, B., Simpson, C., Gottesman, S., \& Hawarden, T. G. 1997, AJ, 114,1427

Márquez, I., Boisson, C., Durret, F., \& Petitjean, P. 1998, A\&A, 333, 459

Marshall, N., Warwick, R. S., \& Pounds, K. A. 1981, MNRAS, 194, 987

Matthews, L. D., Gallagher, J. S., I., \& Van Driel, W. 1999, AJ, 118, 2751

McGaugh, S. S. 1991, ApJ, 380, 140 . 1994, ApJ, 426, 135

Mihos, J. C., \& Bothun, G. D. 1998, ApJ, 500, 619

Mihos, J. C., Bothun, G. D., \& Richstone, D. O. 1993, ApJ, 418, 82

Mirabel, I. F., Dottori, H., \& Lutz, D. 1992, A\&A, 256, L19

Mirabel, I. F., Lutz, D., \& Maza, J. 1991, A\&A, 243, 367

Mirabel, I. F. \& Wilson, A. S. 1984, ApJ, 277, 92

Monet, D. 1996, in BAAS, 188,5404

Papaderos, P., Loose, H. H., Fricke, K. J., \& Thuan, T. X. 1996, A\&A, 314, 59

Papaderos, P., Loose, H. H., Thuan, T. X., \& Fricke, K. J. 1996, A\&AS, 120, 207

Patterson, R. J., \& Thuan, T. X. 1996, ApJS, 107, 103

Prugniel, P., \& Heraudeau, P. 1998, A\&AS, 128, 299

Roberts, M. S. \& Haynes, M. P. 1994, ARA\&A, 32, 115

Rodrigues, I., Dottori, H., Brinks, E., \& Mirabel, I. F. 1999, AJ, 117, 2695

Rownd, B. K., \& Young, J. S. 1999, AJ, 118, 670

Salo, H., \& Laurikainen, E. 1993, ApJ, 410, 586

Sanders, D. B., \& Mirabel, I. F. 1985, ApJ, 298, L31

Schmitt, H. R., Storchi-Bergmann, T., \& Baldwin, J. A. 1994, ApJ, 423, 237

Schombert, J. M., Wallin, J. F., \& Struck-Marcell, C. 1990, AJ, 99, 497

Skillman, E. 1996, in ASP Conf. Ser. 106, The Minnesota Lectures on Extragalactic Neutral Hydrogen, ed. E. Skillman (San Francisco: ASP), 208

Skillman, E. D. 1987, in Star Formation in Galaxies, ed. C. J. Lonsdale \& C. J. Persson (Washington: NASA), 263

Smith, B. J., Struck, C., Kenney, J. D. P., \& Jogee, S. 1999, AJ, 117, 1237

Sofue, Y., Wakamatsu, K. I., Taniguchi, Y., \& Nakai, N. 1993, PASJ, 45, 43

Sotnikova, N. Y. \& Reshetnikov, V. P. 1998, AZh Pisma, 24, 97

Springel, V. 2000, MNRAS, 312, 859

Springel, V., \& White, S. D. M. 1999, MNRAS, 307, 162

Storchi-Bergmann, T., Rodriguez-Ardila, A., Schmitt, H. R., Wilson, A. S., \& Baldwin, J. A. 1996, ApJ, 472, 83

Struck, C. 1999, Phys. Rep., 321, 1

Taylor, C. L., Brinks, E., Pogge, R. W., \& Skillman, E. D. 1994, AJ, 107, 971

Thomasson, M., \& Donner, K. J. 1993, A\&A, 272, 153

Toomre, A., \& Toomre, J. 1972, ApJ, 178, 623

Torres-Peimbert, S., Peimbert, M., \& Fierro, J. 1989, ApJ, 345, 186

Ulvestad, J. S., \& Wilson, A. S. 1984, ApJ, 285, 439

van der Hulst, J. M. 1979, A\&A, 71, 131

van der Hulst, J. M., Skillman, E. D., Smith, T. R., Bothun, G. D., McGaugh, S. S., \& De Blok, W. J. G. 1993, AJ, 106, 548

van Zee, L., Haynes, M. P., Salzer, J. J., \& Broeils, A. H. 1997, AJ, 113, 1618

Wallin, J. F. 1990, AJ, 100, 1477

Weedman, D. W. 1971, Astrophys. Lett., 9, 49

Wehrle, A. E., \& Morris, M. 1988, AJ, 95, 1689

Weilbacher, P., Duc, P.-A., Fritze-von Alvensleben, U., \& Fricke, K. J. 2000, A\&A, 358, 819 